Article

\title{
Novel Insect Antifeedant and Ixodicidal Nootkatone Derivatives
}

\author{
Alberto Galisteo Pretel ${ }^{1}$, Helena Pérez del Pulgar ${ }^{1}$, A. Sonia Olmeda ${ }^{2}$, \\ Azucena Gonzalez-Coloma ${ }^{3}\left(\mathbb{D}\right.$, Alejandro F. Barrero ${ }^{1, *}$ and José Francisco Quílez del Moral ${ }^{1, *}$ (D) \\ 1 Department of Organic Chemistry, Institute of Biotechnology, University of Granada, 18071 Granada, Spain; \\ albertogapre@ugr.es (A.G.P.); helenaperezpv@ugr.es (H.P.d.P.) \\ 2 Faculty of Veterinary, Complutense University of Madrid (UCM), 28040 Madrid, Spain; angeles@ucm.es \\ 3 Institute of Agricultural Sciences, CSIC, 28006, Madrid, Spain; azu@ica.csic.es \\ * Correspondence: afbarre@ugr.es (A.F.B.); jfquilez@ugr.es (J.F.Q.d.M.); \\ Tel.: +34-958243185 (A.F.B. \& J.F.Q.d.M.)
}

Received: 26 September 2019; Accepted: 12 November 2019; Published: 16 November 2019

\begin{abstract}
Naturally occurring nootkatone, with reported insecticidal and acaricidal properties, has been used as a lead to generate molecular diversity and, consequently, new insect antifeedant and ixodicidal compounds. A total of 22 derivatives were generated by subjecting this molecule to several reactions including dehydrogenation with the iodine/DMSO system, oxidation with $\mathrm{SeO}_{2}$, epoxidation with $m \mathrm{CPBA}$, oxidation or carbon homologations of the $\alpha$-carbonyl position with TMSOTf (trimethylsilyl trifluoromethanesulfonate) followed by Rubottom and Dess Martin periodane oxidations, condensation with formaldehyde using $\mathrm{Yb}(\mathrm{OTf})_{3}$ as catalyst and dehydroxilation using the Grieco protocol. The insect antifeedant (against Myzus persicae and Ropaloshysum padi) and ixodicidal (against the tick Hyalomma lusitanicum) activities of these compounds were tested. Compound $\mathbf{2 0}$ was the most active substance against M. persicae and R. padi, and twice more efficient than nootkatone in the antitick test.
\end{abstract}

Keywords: organic synthesis; natural products; pesticides; antiticks; antifeedant

\section{Introduction}

The well-known advantages of biopesticides compared to conventional synthetic pesticides account for the increasing number of research papers published in this field in recent years [1,2]. Conventional pesticides and biopesticides can be categorized according to the origin of their active ingredients, with one of these categories encompassing synthetic natural derived (SND) substances [2]. In this context, we focused our attention on nootkatone (1), a sesquiterpene which was isolated for the first time from the essential oil of the heartwood of Alaska yellow cedar (Cupressus nootkatensis) [3,4], and is also present in plants of Citrus genus [5], Vetiveria species [6] and others natural sources [7]. Our interest in this molecule was based on its very interesting insecticidal and acaricidal properties [4,8-14]. In this sense, formulations acting as pesticides against mosquitoes and ticks are currently being developed [15]. However, the mode of action of this product remain uncertain [16].

Considering the repellent effects and insecticidal activity of nootkatone, we planned to use this renewable and chiral molecule as natural lead to generate molecular diversity with the ultimate goal of obtaining new SND insect antifeedants (against Rhopalosiphum padi, Myzus persicae, Spodoptera littoralis) and ixodicidal (against Hyalomma lusitanicum) compounds. The moth S. littoralis, also known as Egyptian cotton leaf worm, is reported to be a pest of this plant and of a high variety of vegetable crops [17]. The aphid M. persicae is known to infect many species from 40 plant families, with special prevalence in Brassica crops [18]. R. padi is also an aphid and is considered one of the most important 
cereal pest [19]. Hyalomma lusitanicum is a tick mainly found throughout the Mediterranean area. Apart from its widely known detrimental effects on several species of cattle, this pest is an efficient vector transmitter of the Crimean-Congo hemorrhagic fever disease [20].

Nootkatone features an appropriate structure with functionalizations that may allow an easy and efficient synthesis of a series of derivatives following a Diversity Oriented Synthesis (DOS) approach [21]. Thus, the presence of an unsaturated ketone would enable the production of further insaturations at $\mathrm{C} 3-\mathrm{C} 4$ or $\mathrm{C} 8-\mathrm{C}$. Also, the carbonyl group at $\mathrm{C} 2$ should permit the generation of new derivatives functionalized at C3. Additionally, the direct and allylic oxidations of the double bonds present in the molecule will help increase structural diversity (Figure 1).

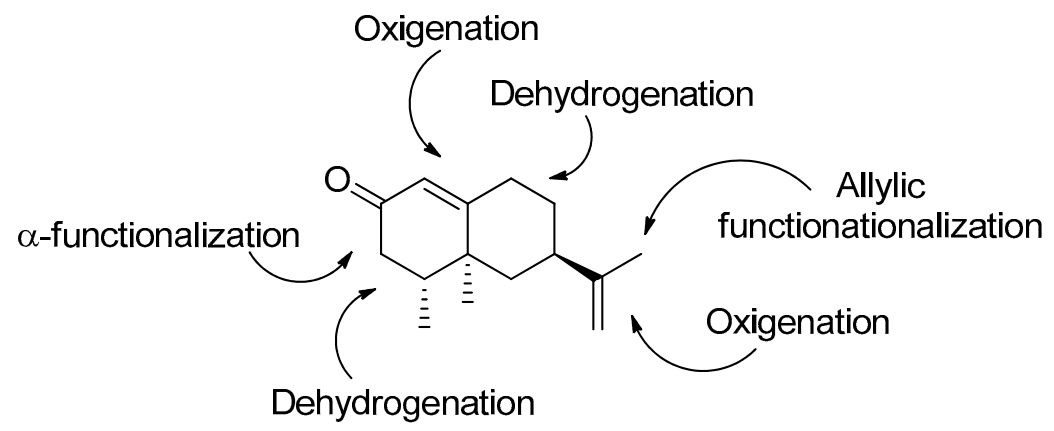

Figure 1. Generation of structural diversity from nootkatone (1).

To improve persistence of biological activity in open areas, new less volatile oxygenated nootkatone derivatives have been designed.

\section{Experimental}

\subsection{Chemicals and Instrument}

Silica gel $60(35-70 \mu \mathrm{m})$ was used for flash column chromatography. NMR spectra (see Supplementary Materials) were obtained in a Varian Direct-Drive $600\left({ }^{1} \mathrm{H} 600 \mathrm{MHz} /{ }^{13} \mathrm{C} 150 \mathrm{MHz}\right)$, Varian Direct-Drive $500\left({ }^{1} \mathrm{H} 500 \mathrm{MHz} /{ }^{13} \mathrm{C} 125 \mathrm{MHz}\right)$ and Varian Direct-Drive $400\left({ }^{1} \mathrm{H} 400 \mathrm{MHz} /{ }^{13} \mathrm{C} 100 \mathrm{MHz}\right)$. Accurate mass determinations were performed on a SYNAPT G2-Si Q-TOF mass spectrometer (Waters, Milford, MA, USA) equipped with high-efficiency T-Wave ion mobility and an orthogonal Z-spray ${ }^{\mathrm{TM}}$ electrospray ionization (ESI) source. MassLynx v.4.1 software was used for HRMS instrument control, peak detection, and integration. Reactions were monitored by TLC on $0.25-\mathrm{mm}$ E. Merck silica gel plates (60F-254) visualized under UV light and by applying a phosphomolybdic acid solution in EtOH followed by heat. High quality reagents were purchased at the highest quality that was commercially available and were used without further purification.

\subsection{Synthesis of Nootkatone Derivatives (See Table 1, Scheme 1)}

\subsubsection{Dehydrogenation of Nootkatone with the System $\mathrm{I}_{2} /$ Dimethysulfoxide (DMSO)}

To a solution of nootkatone $(\mathbf{1}),(219 \mathrm{mg}, 1 \mathrm{mmol})$ in toluene $(10 \mathrm{~mL}), \mathrm{I}_{2}(25 \mathrm{mg}, 0.1 \mathrm{mmol})$ and DMSO (0.34 mL, $5 \mathrm{mmol})$ were added under argon atmosphere. The resulting mixture was refluxed for $6 \mathrm{~h}$. Upon consumption of the starting material, the reaction mixture was diluted with methyl tert-butyl ether (MTBE) $(60 \mathrm{~mL})$, washed with saturated $\mathrm{Na}_{2} \mathrm{~S}_{2} \mathrm{O}_{4}(3 \times 30 \mathrm{~mL})$, brine $(3 \times 30 \mathrm{~mL})$, dried over anhydrous $\mathrm{Na}_{2} \mathrm{SO}_{4}$, and concentrated in vacuo. The crude product was purified by flash chromatography (H:MTBE 4:1) to obtain 2 [22] (31 mg, 14\%), 3 [23] (110 mg, 51\%) and 4 (56 mg, 16\%).

Compound 4: $[\alpha]_{\mathrm{D}}=+34.3(c=1, \mathrm{DCM}) .{ }^{1} \mathrm{H} \mathrm{NMR}\left(600 \mathrm{MHz} \mathrm{CDCl}_{3}\right) \delta 6.23(\mathrm{dd}, J=10.3,2.3 \mathrm{~Hz}$, $1 \mathrm{H}), 6.20(\mathrm{~d}, J=10.3 \mathrm{~Hz}, 1 \mathrm{H}), 5.84(\mathrm{~s}, 1 \mathrm{H}), 4.94(\mathrm{~d}, J=12.5 \mathrm{~Hz}, 1 \mathrm{H}), 4.87(\mathrm{~s}, 1 \mathrm{H}), 4.82(\mathrm{~s}, 1 \mathrm{H}), 3.03(\mathrm{dd}$, $J=11.3,4.6 \mathrm{~Hz}, 1 \mathrm{H}), 2.28(\mathrm{dq}, J=13.2,6.7 \mathrm{~Hz}, 1 \mathrm{H}), 2.03(\mathrm{dd}, J=12.9,4.9 \mathrm{~Hz}, 1 \mathrm{H}), 1.75(\mathrm{~s}, 3 \mathrm{H}), 1.33(\mathrm{t}$, 
$J=12.2 \mathrm{~Hz}, 1 \mathrm{H}), 1.27(\mathrm{~d}, J=6.7 \mathrm{~Hz}, 3 \mathrm{H}), 1.08(\mathrm{~s}, 3 \mathrm{H}) .{ }^{13} \mathrm{C} \mathrm{NMR}\left(151 \mathrm{MHz}, \mathrm{CDCl}_{3}\right) \delta 192.88(\mathrm{C}), 161.97$ (C), $146.81(\mathrm{C}), 141.63(\mathrm{CH}), 127.53(\mathrm{CH}), 121.74(\mathrm{CH}), 112.01\left(\mathrm{CH}_{2}\right), 48.70(\mathrm{CH}), 41.80(\mathrm{CH}), 41.67(\mathrm{CH})$, $39.44\left(\mathrm{CH}_{2}\right), 38.71(\mathrm{C}), 20.47\left(\mathrm{CH}_{3}\right), 17.62\left(\mathrm{CH}_{3}\right), 16.99\left(\mathrm{CH}_{3}\right)$. HRMS TOF (ESI+) $\mathrm{m} / z$ calculated for $\mathrm{C}_{15} \mathrm{H}_{19} \mathrm{IO}[\mathrm{M}+\mathrm{H}]^{+}$342.0475, found 342.0481.

\subsubsection{Dehydrogenation of $\mathbf{1}$ with 2,3-dichloro-5,6-dicyanobenzoquinone (DDQ)}

To a solution of nootkatone $\mathbf{1}(655 \mathrm{mg}, 3 \mathrm{mmol})$ in $20 \mathrm{~mL}$ of benzene, under an argon atmosphere, was added DDQ $(749 \mathrm{mg}, 3.3 \mathrm{mmol})$ and $0.22 \mathrm{~mL}$ of acid acetic. The reaction mixture was stirred for 2 days in reflux. Then, the solution was filtered at room temperature and the solvent was evaporated under low pressure. The crude reaction mixture was then re-dissolved in EtOAc, and washed with saturated $\mathrm{NaHCO}_{3}$ and brine, dried with anhydrous $\mathrm{Na}_{2} \mathrm{SO}_{4}$, and concentrated in vacuo. Purification by flash chromatography (H:MTBE, 1:1) provided $328 \mathrm{mg}$ of 3 [23] (51\%) and $103 \mathrm{mg}$ of $\mathbf{1}$ (16\%).

\subsubsection{Reaction of Nootkatone with $\mathrm{SeO}_{2}$ (See Scheme 2)}

To a solution of nootkatone 1 (216 mg, $0.99 \mathrm{mmol})$ in $\mathrm{EtOH}(10 \mathrm{~mL}), \mathrm{SeO}_{2}(100 \mathrm{mg}, 1 \mathrm{mmol})$ was added and the resulting solution was refluxed for $3 \mathrm{~h}$. Upon consumption of the starting material, the reaction mixture was concentrated in vacuo. The crude reaction mixture was solubilized in EtOAc $(50 \mathrm{~mL})$, washed with saturated $\mathrm{NaHCO}_{3}(3 \times 30 \mathrm{~mL})$, brine $(3 \times 30 \mathrm{~mL})$, dried over anhydrous $\mathrm{Na}_{2} \mathrm{SO}_{4}$, and concentrated in vacuo. Purification by flash chromatography was carried out (H:MTBE, from 4:1 to 1:1) to give $75 \mathrm{mg}$ of 5 [24] (32\%), $28 \mathrm{mg}$ of 6 [24] (11\%) and $35 \mathrm{mg}$ of 7 (15\%).

Compound 7. $[\alpha]_{\mathrm{D}}=-0.39(\mathrm{c}=1, \mathrm{DCM}) .{ }^{1} \mathrm{H}$ NMR $\left(500 \mathrm{MHz}, \mathrm{CDCl}_{3}\right) \delta 4.94(\mathrm{~s}, 1 \mathrm{H}), 4.80(\mathrm{~s}$, $1 \mathrm{H}), 2.69(\mathrm{~d}, J=16.3 \mathrm{~Hz}, 1 \mathrm{H}), 2.56(\mathrm{~d}, J=16.4 \mathrm{~Hz}, 1 \mathrm{H}), 2.26-2.12(\mathrm{~m}, 4 \mathrm{H}), 1.77(\mathrm{~s}, 3 \mathrm{H}), 1.72-1.77(\mathrm{~m}$, $3 \mathrm{H}), 1.54-1.45(\mathrm{~m}, 2 \mathrm{H}), 1.11(\mathrm{~d}, J=0.8 \mathrm{~Hz}, 3 \mathrm{H}), 0.90(\mathrm{~d}, J=5.8 \mathrm{~Hz}, 3 \mathrm{H}) .{ }^{13} \mathrm{C} \mathrm{NMR}\left(126 \mathrm{MHz}, \mathrm{CDCl}_{3}\right)$ $\delta=209.05(\mathrm{C}), 145.62(\mathrm{C}), 109.61\left(\mathrm{CH}_{2}\right), 89.89(\mathrm{C}), 88.66(\mathrm{C}), 49.73\left(\mathrm{CH}_{2}\right), 45.91(\mathrm{C}), 44.66\left(\mathrm{CH}_{2}\right), 44.35$ $\left(\mathrm{CH}_{2}\right), 37.96(\mathrm{CH}), 35.92\left(\mathrm{CH}_{2}\right), 32.85\left(\mathrm{CH}_{2}\right), 18.91\left(\mathrm{CH}_{3}\right), 15.33\left(\mathrm{CH}_{3}\right), 14.27\left(\mathrm{CH}_{3}\right)$. HRMS TOF (ESI+) $m / z$ calculated for $\mathrm{C}_{15} \mathrm{H}_{23} \mathrm{O}_{2}[\mathrm{M}+\mathrm{H}]^{+} 235.1691$, found 235.1698 .

\subsubsection{Acetylation of Compound 5}

To a solution of 5 ( $300 \mathrm{mg}, 1.28 \mathrm{mmol})$ in pyridine $(10 \mathrm{~mL}), 3 \mathrm{mg}$ of 4-dimethhylaminopyridine were added under argon atmosphere and the resulting mixture was heated to reflux temperature. Then $\mathrm{Ac}_{2} \mathrm{O}(1.82 \mathrm{~mL}, 19.2 \mathrm{mmol})$ was added and the mixture refluxed for $1 \mathrm{~h}$. Upon consumption of the starting material, $10 \mathrm{~g}$ of ice and $50 \mathrm{~mL}$ of MTBE were poured over the crude mixture. The organic layer was washed with $2 \mathrm{~N} \mathrm{HCl}(3 \times 30 \mathrm{~mL})$, saturated $\mathrm{NaHCO}_{3}(3 \times 30 \mathrm{~mL})$ and brine $(3 \times 30 \mathrm{~mL})$, dried over anhydrous $\mathrm{Na}_{2} \mathrm{SO}_{4}$, and concentrated in vacuo. The crude product was purified by flash chromatography (H:MTBE, 1:2) to give $291 \mathrm{mg}$ of $8(82 \%)$.

Compound 8. $[\alpha]_{\mathrm{D}}=+99.8(c=1, \mathrm{DCM}) .{ }^{1} \mathrm{H}$ NMR $\left(400 \mathrm{MHz}, \mathrm{CDCl}_{3}\right) \delta 5.75(\mathrm{~s}, 1 \mathrm{H}), 5.07(\mathrm{~s}, 1 \mathrm{H})$, $4.96(\mathrm{~s}, 1 \mathrm{H}), 4.56(\mathrm{~s}, 2 \mathrm{H}), 2.50(\mathrm{dddd}, J=15.6,13.7,5.1,2.0 \mathrm{~Hz}, 1 \mathrm{H}), 2.43-2.29(\mathrm{~m}, 2 \mathrm{H}), 2.29-2.16(\mathrm{~m}$, $2 \mathrm{H}), 2.08(\mathrm{~s}, 3 \mathrm{H}), 2.06-1.87(\mathrm{~m}, 3 \mathrm{H}), 1.35(\mathrm{dd}, J=13.6,12.6,4.2 \mathrm{~Hz}, 1 \mathrm{H}), 1.25-1.10(\mathrm{~m}, 1 \mathrm{H}), 1.09(\mathrm{~s}, 3 \mathrm{H})$, $0.94(\mathrm{~d}, J=6.8 \mathrm{~Hz}, 3 \mathrm{H}) \cdot{ }^{13} \mathrm{C}$ NMR $\left(101 \mathrm{MHz}, \mathrm{CDCl}_{3}\right) \delta=199.35(\mathrm{C}), 170.64(\mathrm{C}), 169.63(\mathrm{C}), 147.34(\mathrm{C})$, $124.83\left(\mathrm{CH}_{2}\right), 111.83(\mathrm{CH}), 66.01\left(\mathrm{CH}_{2}\right), 44.15\left(\mathrm{CH}_{2}\right), 42.00\left(\mathrm{CH}_{2}\right), 40.36(\mathrm{CH}), 39.33(\mathrm{C}), 36.37(\mathrm{CH})$, $32.90\left(\mathrm{CH}_{2}\right), 31.74\left(\mathrm{CH}_{2}\right), 20.93\left(\mathrm{CH}_{3}\right), 16.70\left(\mathrm{CH}_{3}\right), 14.85\left(\mathrm{CH}_{3}\right)$. HRMS TOF $(\mathrm{ES}+) \mathrm{m} / z$ calculated for $\mathrm{C}_{17} \mathrm{H}_{25} \mathrm{O}_{3}[\mathrm{M}+\mathrm{H}]+277.1804$, found 277.1831.

\subsubsection{Reaction of Compound 3 with $\mathrm{SeO}_{2}$ (See Scheme 3)}

To a solution of 3 (115 mg, $0.53 \mathrm{mmol})$ in $\mathrm{EtOH}(5 \mathrm{~mL}), \mathrm{SeO}_{2}(96 \mathrm{mg}, 0.86 \mathrm{mmol})$ was added and the resulting solution was the refluxed for $3 \mathrm{~h}$. The reaction mixture was then concentrated in vacuo, re-dissolved in EtOAc $(30 \mathrm{~mL})$, washed with saturated $\mathrm{NaHCO}_{3}(3 \times 20 \mathrm{~mL})$, brine $(3 \times 20 \mathrm{~mL})$, dried over anhydrous $\mathrm{Na}_{2} \mathrm{SO}_{4}$, and concentrated in vacuo. The crude product was purified by flash chromatography (H:MTBE, from 1:2 to 1:4) to obtain $30 \mathrm{mg}$ of $\mathbf{3}(26 \%), 65 \mathrm{mg}$ of $\mathbf{9}(53 \%)$ and $17 \mathrm{mg}$ of $10(14 \%)$. 
Compound 9. $[\alpha]_{\mathrm{D}}=+29.6(c=1, \mathrm{DCM}) .{ }^{1} \mathrm{H}$ NMR $\left(500 \mathrm{MHz}, \mathrm{CDCl}_{3}\right) \delta=6.11(\mathrm{~s}, 2 \mathrm{H}), 5.10(\mathrm{~s}, 1 \mathrm{H})$, $4.90(\mathrm{~s}, 1 \mathrm{H}), 4.16(\mathrm{~s}, 2 \mathrm{H}), 2.71-2.54(\mathrm{~m}, 2 \mathrm{H}), 2.50$ (dddd, $J=15.6,13.7,5.1,2.0 \mathrm{~Hz}, 1 \mathrm{H}), 2.18-2.03(\mathrm{~m}$, $2 \mathrm{H}), 2.01(\mathrm{~s}, 3 \mathrm{H}), 1.47-1.36(\mathrm{~m}, 1 \mathrm{H}), 1.36(\mathrm{~s}, 3 \mathrm{H}), 1.28(\mathrm{t}, J=12.8 \mathrm{~Hz}, 1 \mathrm{H}) .{ }^{13} \mathrm{C} \mathrm{NMR}(126 \mathrm{MHz}, \mathrm{CDCl} 3)$ $\delta=186.39(\mathrm{C}), 167.10(\mathrm{C}), 165.66(\mathrm{C}), 151.57(\mathrm{C}), 126.78(\mathrm{CH}), 124.61(\mathrm{CH}), 109.82\left(\mathrm{CH}_{2}\right), 65.30\left(\mathrm{CH}_{2}\right)$, $43.46(\mathrm{C}), 42.40\left(\mathrm{CH}_{2}\right), 35.72(\mathrm{CH}), 33.90\left(\mathrm{CH}_{2}\right), 32.85\left(\mathrm{CH}_{2}\right), 23.24\left(\mathrm{CH}_{3}\right), 18.86\left(\mathrm{CH}_{3}\right)$. HRMS TOF (ESI+) $m / z$ calculated for $\mathrm{C}_{15} \mathrm{H}_{21} \mathrm{O}_{2}[\mathrm{M}+\mathrm{H}]^{+} 233.1542$, found 233.1671 .

Compound 10. $[\alpha]_{\mathrm{D}}=+40.6(c=1, \mathrm{DCM}) .{ }^{1} \mathrm{H}$ NMR $\left(500 \mathrm{MHz}, \mathrm{CD}_{3} \mathrm{COCD}_{3}\right) \delta 9.59(\mathrm{~s}, 1 \mathrm{H}), 6.42$ $(\mathrm{d}, J=1.1 \mathrm{~Hz}, 1 \mathrm{H}), 6.18(\mathrm{~s}, 1 \mathrm{H}), 6.00(\mathrm{dt}, J=4.8,1.7 \mathrm{~Hz}, 2 \mathrm{H}), 3.12(\mathrm{ttd}, J=12.7,3.5,1.1 \mathrm{~Hz}, 1 \mathrm{H}), 2.77$ (ddd, $J=13.8,4.9,1.7 \mathrm{~Hz}, 1 \mathrm{H}), 2.46(\mathrm{ddd}, J=13.9,4.2,2.6 \mathrm{~Hz}, 1 \mathrm{H}), 2.16(\mathrm{ddd}, J=12.7,3.5,2.3 \mathrm{~Hz}$, 1H), 2.05-1.99 (m, 4H), $1.44(\mathrm{~s}, 3 \mathrm{H}), 1.43-1.37(\mathrm{~m}, 1 \mathrm{H}), 1.26(\mathrm{t}, J=12.7 \mathrm{~Hz}, 1 \mathrm{H}) .{ }^{13} \mathrm{C} \mathrm{NMR}(126 \mathrm{MHz}$, $\left.\mathrm{CD}_{3} \mathrm{COCD}_{3}\right) \delta 194.04(\mathrm{CH}), 184.78(\mathrm{C}), 166.14(\mathrm{C}), 164.92(\mathrm{C}), 153.28(\mathrm{C}), 133.45\left(\mathrm{CH}_{2}\right), 126.35(\mathrm{CH})$, 124.30 (CH), $43.25(\mathrm{C}), 41.72\left(\mathrm{CH}_{2}\right), 33.16\left(\mathrm{CH}_{2}\right), 32.23\left(\mathrm{CH}_{2}\right), 30.77(\mathrm{CH}), 22.66\left(\mathrm{CH}_{3}\right), 17.89\left(\mathrm{CH}_{3}\right)$. HRMS TOF (ESI+) $m / z$ calculated for $\mathrm{C}_{15} \mathrm{H}_{19} \mathrm{O}_{2}[\mathrm{M}+\mathrm{H}]^{+}$231.1382, found 231.1385.

\subsubsection{Reaction of Nootkatone with $m$ chloroperbenzoic Acid ( $m$ CPBA) (See Scheme 4)}

To a solution of nootkatone (1) $(220 \mathrm{mg}, 1 \mathrm{mmol})$ in DCM (6 mL), mCPBA (595 mg, $3.4 \mathrm{mmol})$ was added and stirred at room temperature for 5 days. Upon consumption of the starting material, the reaction mixture was diluted with MTBE $(50 \mathrm{~mL})$, washed with $10 \% \mathrm{Na}_{2} \mathrm{SO}_{3}(3 \times 30 \mathrm{~mL}), 10 \%$ $\mathrm{NaOH}(3 \times 30 \mathrm{~mL})$ and brine $(3 \times 30 \mathrm{~mL})$, dried over anhydrous $\mathrm{Na}_{2} \mathrm{SO}_{4}$, and concentrated in vacuo. The crude product was purified by flash chromatography (H:MTBE, 3:1) to obtain 49 mg of $\mathbf{1 1}$ [25] (21\%), $58 \mathrm{mg}$ of $\mathbf{1 2}$ [26] (23\%) and $79 \mathrm{mg}$ of $\mathbf{1 3}(30 \%)$.

Compound 13. $[\alpha]_{\mathrm{D}}=+5.5(\mathrm{c}=1, \mathrm{DCM}) .{ }^{1} \mathrm{H} \mathrm{NMR}\left(400 \mathrm{MHz}, \mathrm{CDCl}_{3}\right) \delta 4.78(\mathrm{~s}, 2 \mathrm{H}), 2.89(\mathrm{~m}, 2 \mathrm{H})$, 2.64-2.52 (m, 4H), 2.43-2.37 (m, 2H), 1.92-1.74 (m, 8H), 1.67-1.44 (m, 2H), 1.38-1.15 (m, 6H), $1.25(\mathrm{~s}$, $3 \mathrm{H}), 1.24(\mathrm{~s}, 3 \mathrm{H}), 1.08(\mathrm{~s}, 3 \mathrm{H}), 1.08(\mathrm{~s}, 3 \mathrm{H}), 0.99(\mathrm{~d}, J=7.2, \mathrm{~Hz}, 3 \mathrm{H}), 0.98(\mathrm{~d}, J=7.2, \mathrm{~Hz}, 3 \mathrm{H}) .{ }^{13} \mathrm{C} \mathrm{NMR}$ $\left(101 \mathrm{MHz}, \mathrm{CDCl}_{3}\right) \delta=170.35,170.31(\mathrm{C}), 86.80,86.68(\mathrm{CH}), 69.13,69.05(\mathrm{C}), 58.76,58.59(\mathrm{C}), 53.50,52.87$ $\left(\mathrm{CH}_{2}\right), 40.60,40.37\left(\mathrm{CH}_{2}\right), 38.65\left(\mathrm{CH}_{2}\right), 38.37,37.87(\mathrm{CH}), 38.23(\mathrm{C}), 37.64,37.62(\mathrm{CH}), 30.78,30.61\left(\mathrm{CH}_{2}\right)$, 26.21, $25.73\left(\mathrm{CH}_{2}\right), 18.54,17.92\left(\mathrm{CH}_{3}\right), 17.12,17.07\left(\mathrm{CH}_{3}\right), 16.60,16.58\left(\mathrm{CH}_{3}\right)$. HRMS TOF (ES+) $\mathrm{m} / \mathrm{z}$ calculated for $\mathrm{C}_{15} \mathrm{H}_{23} \mathrm{O}_{4}[\mathrm{M}+\mathrm{H}]^{+}$267.1583, found 267.1596.

\subsubsection{Synthesis of Compounds 14 and 15 (See Scheme 5)}

To a solution of nootkatone $\mathbf{1}(0.46 \mathrm{~mL}, 2 \mathrm{mmol})$ in dry toluene $(9 \mathrm{~mL})$ at $-78^{\circ} \mathrm{C}, 2.5 \mathrm{~mL}$ of collidine were added and stirred for $15 \mathrm{~min}$, then TMSOTf $(0.9 \mathrm{~mL}, 5 \mathrm{mmol})$ was added and the resulting solution was stirred for additional $10 \mathrm{~min}$. Upon consumption of the starting material, the reaction mixture was diluted with hexane $(15 \mathrm{~mL})$, washed with brine $(3 \times 10 \mathrm{~mL})$ and dried over anhydrous $\mathrm{Na}_{2} \mathrm{SO}_{4}$ and concentrated in vacuo. The crude product was solved in dry DCM $(30 \mathrm{~mL})$ at $0{ }^{\circ} \mathrm{C}$ and then $\mathrm{NaHCO}_{3}$ ( $672 \mathrm{mg}, 8 \mathrm{mmol}$ ) and $m \mathrm{CPBA}(592 \mathrm{mg}, 2,4 \mathrm{mmol}$ ) were added. The resulting mixture was stirred for 3 h. The reaction mixture was then diluted with DCM $(5 \mathrm{~mL})$ filtered through Celite $囚$ and concentrated in vacuo. The crude product was purified using mixtures of eluents of increasing polarity to obtain $30 \mathrm{mg}$ of $\mathbf{1}$ (7\%) (H:MTBE 9:1), $76 \mathrm{mg}$ of $\mathbf{1 4}$ (16\%) (H:MTBE, 5:1) and $77 \mathrm{mg}$ of $\mathbf{1 5}$ (16\%) (H:MTBE,5:1).

Compound 14. $[\alpha]_{D}=+137.8(c=1, \mathrm{DCM}) .{ }^{1} \mathrm{H}$ NMR $\left(600 \mathrm{MHz}, \mathrm{CDCl}_{3}\right) \delta=5.85(\mathrm{~s}, 1 \mathrm{H}), 4.74(\mathrm{~s}$, $1 \mathrm{H}), 4.70(\mathrm{~s}, 1 \mathrm{H}), 3.96(\mathrm{~d}, J=12.7 \mathrm{~Hz}, 1 \mathrm{H}), 2.52(\mathrm{td}, J=13.7,4.5 \mathrm{~Hz}, 1 \mathrm{H}), 2.40(\mathrm{ddd}, J=14.9,4.2,2.6 \mathrm{~Hz}$, $1 \mathrm{H}), 2.34(\mathrm{tt}, J=12.5,3.3 \mathrm{~Hz}, 1 \mathrm{H}), 1.97(\mathrm{dt}, J=13.1,2.9 \mathrm{~Hz}, 1 \mathrm{H}), 1.91-1.95(\mathrm{~m}, 1 \mathrm{H}), 1.82(\mathrm{dq}, J=13.2,6.7$ $\mathrm{Hz}, 1 \mathrm{H}), 1.72(\mathrm{~s}, 3 \mathrm{H}), 1.36(\mathrm{qd}, J=12.9,4.2 \mathrm{~Hz}, 1 \mathrm{H}), 1.22(\mathrm{~s}, 3 \mathrm{H}), 1.14(\mathrm{~d}, J=6.7 \mathrm{~Hz}, 3 \mathrm{H}) .{ }^{13} \mathrm{C} \mathrm{NMR}(151$ $\left.\mathrm{MHz}, \mathrm{CDCl}_{3}\right) \delta=199.65(\mathrm{C}), 172.08(\mathrm{C}), 148.63(\mathrm{C}), 120.98(\mathrm{CH}), 109.46\left(\mathrm{CH}_{2}\right), 73.40(\mathrm{CH}), 47.63(\mathrm{CH})$, $44.18\left(\mathrm{CH}_{2}\right), 40.92(\mathrm{C}), 39.61(\mathrm{CH}), 33.14\left(\mathrm{CH}_{2}\right), 31.75\left(\mathrm{CH}_{2}\right), 20.75\left(\mathrm{CH}_{3}\right), 17.79\left(\mathrm{CH}_{3}\right), 10.81\left(\mathrm{CH}_{3}\right)$. HRMS TOF (ESI+) $m / z$ calculated for $\mathrm{C}_{15} \mathrm{H}_{23} \mathrm{O}_{2}[\mathrm{M}+\mathrm{H}]^{+} 235.1689$, found 235.1698.

Compound 15. $[\alpha]_{\mathrm{D}}=-49.8(c=1, \mathrm{DCM}) .{ }^{1} \mathrm{H} \mathrm{NMR}\left(400 \mathrm{MHz}, \mathrm{CDCl}_{3}\right) \delta=5.82(\mathrm{~s}, 1 \mathrm{H}), 4.72(\mathrm{~s}, 1 \mathrm{H})$, $4.70(\mathrm{~s}, 1 \mathrm{H}), 4.36(\mathrm{~d}, J=5.2 \mathrm{~Hz}, 1 \mathrm{H}), 2.62-2.46(\mathrm{~m}, 2 \mathrm{H}), 2.27(\mathrm{ddd}, J=12.7,4.3,2.7 \mathrm{~Hz}, 1 \mathrm{H}), 2.07-1.96(\mathrm{~m}$, $2 \mathrm{H}), 1.83-1.71(\mathrm{~m}, 2 \mathrm{H}), 1.70(\mathrm{~s}, 3 \mathrm{H}), 1.37-1.28(\mathrm{~m}, 1 \mathrm{H}), 1.26(\mathrm{~s}, 3 \mathrm{H}), 0.91(\mathrm{~d}, J=7.0 \mathrm{~Hz}, 3 \mathrm{H}) .{ }^{13} \mathrm{C} \mathrm{NMR}$ $\left(151 \mathrm{MHz}, \mathrm{CDCl}_{3}\right) \delta=199.59(\mathrm{C}), 170.58(\mathrm{C}), 148.13(\mathrm{C}), 119.46(\mathrm{CH}), 109.69\left(\mathrm{CH}_{2}\right), 73.23(\mathrm{CH}), 43.87$ 
$(\mathrm{CH}), 43.58\left(\mathrm{CH}_{2}\right), 41.11(\mathrm{C}), 39.91(\mathrm{CH}), 34.37\left(\mathrm{CH}_{2}\right), 32.98\left(\mathrm{CH}_{2}\right), 22.54\left(\mathrm{CH}_{3}\right), 20.93\left(\mathrm{CH}_{3}\right), 8.86\left(\mathrm{CH}_{3}\right)$. HRMS TOF (ESI+) $m / z$ calculated for $\mathrm{C}_{15} \mathrm{H}_{23} \mathrm{O}_{2}[\mathrm{M}+\mathrm{H}]^{+} 235.1689$, found 235.1709.

\subsubsection{Synthesis of Compounds $\mathbf{1 6}$ and $\mathbf{1 7}$}

To a solution of the compounds 14 and 15 (40 mg, $0.17 \mathrm{mmol})$ dry DCM (1 mL), DMP (89 mg, $0.21 \mathrm{mmol}$ ) was added under argon atmosphere. The mixture was stirred for $90 \mathrm{~min}$. Then, a saturated solution of $\mathrm{Na}_{2} \mathrm{~S}_{2} \mathrm{O}_{3}-\mathrm{NaHCO}_{3}(3 \mathrm{~mL})$ was added and the resulting mixture was stirred for 30 additional $\mathrm{min}$. Upon consumption of the starting material, the reaction mixture was diluted in MTBE $(10 \mathrm{~mL})$, washed with $\mathrm{NaHCO}_{3}(3 \times 7 \mathrm{~mL})$ and brine $(3 \times 7 \mathrm{~mL})$, dried with anhydrous $\mathrm{Na}_{2} \mathrm{SO}_{4}$, and concentrated in vacuo. Purification by flash chromatography (H:TMBE, 3:1) gave $10 \mathrm{mg}$ of $\mathbf{1 6}(23 \%)$ and $6 \mathrm{mg}$ of $\mathbf{1 7}$ $(14 \%)$.

Compound 16. $[\alpha]_{\mathrm{D}}=-10.4(c=1, \mathrm{DCM}) .{ }^{1} \mathrm{H}$ NMR $\left(400 \mathrm{MHz}, \mathrm{CDCl}_{3}\right) \delta=6.47(\mathrm{~s}, 1 \mathrm{H}), 6.22(\mathrm{~s}$, $1 \mathrm{H}), 4.76(\mathrm{~s}, 1 \mathrm{H}), 4.74(\mathrm{~s}, 1 \mathrm{H}), 2.63(\mathrm{td}, J=13.8,5.0 \mathrm{~Hz}, 1 \mathrm{H}), 2.51-2.41(\mathrm{~m}, 2 \mathrm{H}), 2.11(\mathrm{dt}, J=13.0,3.0 \mathrm{~Hz}$, $1 \mathrm{H}), 2.08-2.01(\mathrm{~m}, 1 \mathrm{H}), 1.97(\mathrm{~s}, 3 \mathrm{H}), 1.72(\mathrm{~s}, 3 \mathrm{H}), 1.41-1.35(\mathrm{~m}, 1 \mathrm{H}), 1.34(\mathrm{~s}, 3 \mathrm{H}), 1.20(\mathrm{t}, J=12.8 \mathrm{~Hz}, 1 \mathrm{H})$. ${ }^{13} \mathrm{C}$ NMR $\left(126 \mathrm{MHz}, \mathrm{CDCl}_{3}\right) \delta=180.44(\mathrm{C}), 170.50(\mathrm{C}), 147.92(\mathrm{C}), 143.85(\mathrm{C}), 134.29(\mathrm{C}), 121.51(\mathrm{CH})$, $110.01\left(\mathrm{CH}_{2}\right), 43.50(\mathrm{C}), 42.53\left(\mathrm{CH}_{2}\right), 39.87(\mathrm{CH}), 33.63\left(\mathrm{CH}_{2}\right), 32.98\left(\mathrm{CH}_{2}\right), 23.220\left(\mathrm{CH}_{3}\right), 20.66\left(\mathrm{CH}_{3}\right)$, $10.81\left(\mathrm{CH}_{3}\right)$. HRMS TOF (ES+) $\mathrm{m} / z$ calculated for $\mathrm{C}_{15} \mathrm{H}_{21} \mathrm{O}_{2}[\mathrm{M}+\mathrm{H}]^{+} 233.1542$, found 233.1531.

Compound 17. $[\alpha]_{\mathrm{D}}=+105.2(c=1, \mathrm{DCM}) .{ }^{1} \mathrm{H} \mathrm{NMR}\left(400 \mathrm{MHz}, \mathrm{CDCl}_{3}\right) \delta=6.19(\mathrm{~s}, 1 \mathrm{H}), 4.79(\mathrm{~s}$, 1H), $4.77(\mathrm{~s}, 1 \mathrm{H}), 2.63(\mathrm{dddd}, J=15.2,13.3,5.1,1.9 \mathrm{~Hz}, 1 \mathrm{H}), 2.55(\mathrm{ddd}, J=14.9,4.4,2.6 \mathrm{~Hz}, 1 \mathrm{H}), 2.41$ (tt, $J=12.6,3.5 \mathrm{~Hz}, 1 \mathrm{H}), 2.06-1.97(\mathrm{~m}, 1 \mathrm{H}), 1.89(\mathrm{t}, J=12.9 \mathrm{~Hz}, 1 \mathrm{H}), 1.84-1.77(\mathrm{~m}, 1 \mathrm{H}), 1.76(\mathrm{~s}, 3 \mathrm{H}), 1.54-1.43$ $(\mathrm{m}, 1 \mathrm{H}), 1.42(\mathrm{~s}, 3 \mathrm{H}), 1.29(\mathrm{~s}, 3 \mathrm{H}) .{ }^{13} \mathrm{C}$ NMR $\left(126 \mathrm{MHz}, \mathrm{CDCl}_{3}\right) \delta=197.15(\mathrm{C}), 186.11(\mathrm{C}), 172.62(\mathrm{C})$, $148.08(\mathrm{C}), 124.92(\mathrm{CH}), 110.03\left(\mathrm{CH}_{2}\right), 81.64(\mathrm{C}), 48.43(\mathrm{C}), 39.41(\mathrm{CH}), 37.48\left(\mathrm{CH}_{2}\right), 33.69\left(\mathrm{CH}_{2}\right), 31.89$ $\left(\mathrm{CH}_{2}\right), 22.59\left(\mathrm{CH}_{3}\right), 20.74\left(\mathrm{CH}_{3}\right), 17.11\left(\mathrm{CH}_{3}\right)$. HRMS TOF (ESI+) $\mathrm{m} / \mathrm{z}$ calculated for $\mathrm{C}_{15} \mathrm{H}_{21} \mathrm{O}_{3}[\mathrm{M}+\mathrm{H}]^{+}$ 249.1491, found 249.1483.

\subsubsection{Synthesis of Compounds 18 and 19 (See Scheme 6)}

To a solution of nootkatone $\mathbf{1}(0.23 \mathrm{~mL}, 1 \mathrm{mmol})$ in dry toluene $(4 \mathrm{~mL})$ cooled at $-78^{\circ} \mathrm{C}, 1.25 \mathrm{~mL}$ of collidine were added and the resulting mixture was stirred for $15 \mathrm{~min}$, then TMSOTf $(0.45 \mathrm{~mL}$, $2.5 \mathrm{mmol}$ ) was added and stirred for 10 additional min. Upon consumption of the starting material, the reaction mixture was diluted with hexane $(15 \mathrm{~mL})$, washed with brine $(3 \times 10 \mathrm{~mL})$ and dried over anhydrous $\mathrm{Na} 2 \mathrm{SO} 4$ and concentrated in vacuo. The crude mixture was dissolved in THF $(10 \mathrm{~mL})$, then $4 \mathrm{~mL}$ of formaldehyde $(37 \%$, water $)$ and $\mathrm{Yb}(\mathrm{OTf})_{3}(550 \mathrm{mg}, 0.9 \mathrm{mmol})$ were added under argon atmosphere and stirred for $24 \mathrm{~h}$. The reaction mixture was then diluted with a solution of saturated $\mathrm{NaHCO}_{3}(60 \mathrm{~mL})$ and extracted with EtOAc $(3 \times 15 \mathrm{~mL})$. The organic layer was washed with $1 \mathrm{~N} \mathrm{HCl}$ $(3 \times 15 \mathrm{~mL})$, brine $(3 \times 15 \mathrm{~mL})$, dried with anhydrous $\mathrm{Na}_{2} \mathrm{SO}_{4}$ and concentrated in vacuo. The crude reaction mixture was purified by column chromatography using mixtures of solvents with increasing polarity to obtain $126 \mathrm{mg}$ of $\mathbf{1}$ (54.7\%) (H:MTBE, 9:1), $86 \mathrm{mg}$ of $\mathbf{1 8}$ (34\%) (H:MTBE, 2:1).and $30 \mathrm{mg}$ of 19 (11\%) (H:MTBE, 2:1).

Compound 18. $[\alpha]_{D}=+118.0(c=1, \mathrm{DCM}) .{ }^{1} \mathrm{H}$ NMR $\left(400 \mathrm{MHz}, \mathrm{CDCl}_{3}\right) \delta=5.77(\mathrm{~d}, J=1.6 \mathrm{~Hz}$, 1H), $4.73(\mathrm{bs}, 1 \mathrm{H}), 4.70(\mathrm{bs}, 1 \mathrm{H}), 4.04(\mathrm{dd}, J=11.2,3.1 \mathrm{~Hz}, 1 \mathrm{H}), 3.72(\mathrm{dd}, J=11.4,6.7 \mathrm{~Hz}, 1 \mathrm{H}), 2.49$ (dddd, $J=15.6,14.0,5.2,1.9 \mathrm{~Hz}, 1 \mathrm{H}), 2.38-2.28(\mathrm{~m}, 3 \mathrm{H}), 2.00(\mathrm{dt}, J=13.1,2.8 \mathrm{~Hz}, 1 \mathrm{H}), 1.94-1.88(\mathrm{~m}, 2 \mathrm{H}), 1.71(\mathrm{~s}$, $3 \mathrm{H}), 1.33(\mathrm{qd}, J=12.7,4.1 \mathrm{~Hz}, 1 \mathrm{H}), 1.14(\mathrm{~s}, 3 \mathrm{H}), 1.11(\mathrm{t}, J=13.0 \mathrm{~Hz}, 1 \mathrm{H}), 0.99(\mathrm{~d}, J=6.8 \mathrm{~Hz}, 3 \mathrm{H}) .{ }^{13} \mathrm{C}$ NMR $\left(126 \mathrm{MHz}, \mathrm{CDCl}_{3}\right) \delta=202.50(\mathrm{C}), 170.84(\mathrm{C}), 148.86(\mathrm{C}), 124.17(\mathrm{CH}), 109.39\left(\mathrm{CH}_{2}\right), 60.39\left(\mathrm{CH}_{2}\right), 49.18$ $(\mathrm{CH}), 44.04\left(\mathrm{CH}_{2}\right), 41.27(\mathrm{CH}), 40.26(\mathrm{CH}), 39.79(\mathrm{C}), 33.05\left(\mathrm{CH}_{2}\right), 31.60\left(\mathrm{CH}_{2}\right), 20.73\left(\mathrm{CH}_{3}\right), 17.25\left(\mathrm{CH}_{3}\right)$, $12.12\left(\mathrm{CH}_{3}\right)$. HRMS TOF (ESI+) $\mathrm{m} / z$ calculated for $\mathrm{C}_{16} \mathrm{H}_{25} \mathrm{O}_{2}[\mathrm{M}+\mathrm{H}]^{+} 249.1855$, found 249.1843 .

Compound 19. $[\alpha]_{\mathrm{D}}=+238.7(c=1, \mathrm{DCM}) .{ }^{1} \mathrm{H}$ NMR $\left(400 \mathrm{MHz}, \mathrm{CDCl}_{3}\right) \delta 5.54(\mathrm{dd}, J=5.2,2.3 \mathrm{~Hz}$, 1H), $4.77(\mathrm{bs}, 1 \mathrm{H}), 4.74(\mathrm{bs}, 1 \mathrm{H}), 4.04(\mathrm{~d}, J=11.7 \mathrm{~Hz}, 1 \mathrm{H}), 3.99(\mathrm{~d}, J=11.5 \mathrm{~Hz}, 1 \mathrm{H}), 3.93(\mathrm{~d}, J=11.5 \mathrm{~Hz}$, $1 \mathrm{H}), 3.72(\mathrm{~d}, J=11.7 \mathrm{~Hz}, 1 \mathrm{H}), 2.79(\mathrm{dd}, J=18.6,7.8 \mathrm{~Hz}, 1 \mathrm{H}), 2.34-2.05(\mathrm{~m}, 4 \mathrm{H}), 1.95(\mathrm{ddd}, J=17.6,11.2$, $2.3 \mathrm{~Hz}, 1 \mathrm{H}), 1.75(\mathrm{~s}, 3 \mathrm{H}), 1.75-1.70(\mathrm{~m}, 1 \mathrm{H}), 1.22(7, J=12.5 \mathrm{~Hz}, 1 \mathrm{H}), 0.95(\mathrm{~d}, J=6.6 \mathrm{~Hz}, 3 \mathrm{H}), 0.86(\mathrm{~s}$, 3H). ${ }^{13} \mathrm{C}$ NMR $\left(126 \mathrm{MHz}, \mathrm{CDCl}_{3}\right) \delta 218.09$ (C), 149.25 (C), $142.18(\mathrm{C}), 122.99(\mathrm{CH}), 109.09\left(\mathrm{CH}_{2}\right), 70.60(2$ 
x $\left.\mathrm{CH}_{2}\right), 57.81(\mathrm{C}), 44.22\left(\mathrm{CH}_{2}\right), 41.14\left(\mathrm{CH}_{2}\right), 38.08(\mathrm{C}), 37.28(\mathrm{CH}), 36.62(\mathrm{CH}), 31.18\left(\mathrm{CH}_{2}\right), 20.71\left(\mathrm{CH}_{3}\right)$, $17.82\left(\mathrm{CH}_{3}\right), 15.26\left(\mathrm{CH}_{3}\right)$. HRMS TOF (ESI+) $\mathrm{m} / z$ calculated for $\mathrm{C}_{17} \mathrm{H}_{27} \mathrm{O}_{3}[\mathrm{M}+\mathrm{H}]^{+} 279.1960$, found 279.1950 .

\subsubsection{Synthesis of Compound 20}

To a solution of $18(75 \mathrm{mg}, 0.3 \mathrm{mmol})$ in dry TFH $(2.5 \mathrm{~mL}), 0.13 \mathrm{~mL}$ of $n$-Bu $\mathrm{P}(0.46 \mathrm{mmol})$ and $1.3 \mathrm{mg}$ of $o-\mathrm{NO}_{2} \mathrm{PhSeCN}(0.46 \mathrm{mmol})$ were added under argon atmosphere and the resulting solution was stirred for $80 \mathrm{~min}$ at room temperature. Upon consumption of starting material, the reaction mixture was diluted with MTBE $(10 \mathrm{~mL})$, was washed with saturated $\mathrm{NH}_{4} \mathrm{Cl}(3 \times 5 \mathrm{~mL})$, brine $(3 \times$ $5 \mathrm{~mL}$ ), dried with anhydrous $\mathrm{Na}_{2} \mathrm{SO}_{4}$, and concentrated in vacuo. The crude reaction mixture was dissolved in THF $(30 \mathrm{ml})$ and $30 \% \mathrm{H}_{2} \mathrm{O}_{2}(0.06 \mathrm{~mL}, 0.48 \mathrm{mmol})$ was added under argon atmosphere. The resulting mixture was stirred for $15 \mathrm{~min}$ at room temperature. Upon consumption of the starting material, the reaction mixture was diluted with MTBE $(30 \mathrm{~mL})$, washed with brine $(3 \times 20 \mathrm{~mL})$, dried with anhydrous $\mathrm{Na}_{2} \mathrm{SO}_{4}$, and concentrated in vacuo and purified by flash chromatography (H: MTBE, 1:2) to obtain $48 \mathrm{mg}$ of $20(71 \%)$.

Compound 20. $[\alpha]_{\mathrm{D}}=+149.2(c=1, \mathrm{DCM}) .{ }^{1} \mathrm{H} \mathrm{NMR}\left(400 \mathrm{MHz}, \mathrm{CD}_{3} \mathrm{COCD}_{3}\right) \delta 5.92(\mathrm{~s}, 1 \mathrm{H}), 5.80$ $(\mathrm{s}, 1 \mathrm{H}), 5.22(\mathrm{~s}, 1 \mathrm{H}), 4.75(\mathrm{bs}, 1 \mathrm{H}), 4.73(\mathrm{bs}, 1 \mathrm{H}), 2.67-2.53(\mathrm{~m}, 2 \mathrm{H}), 2.44(\mathrm{ddd}, J=15.3,4.1,2.8 \mathrm{~Hz}, 1 \mathrm{H})$, $2.37(\mathrm{tt}, J=12.6,3.3 \mathrm{~Hz}, 1 \mathrm{H}), 2.03-1.98(\mathrm{~m}, 1 \mathrm{H}), 1.95-1.87(\mathrm{~m}, 1 \mathrm{H}), 1.75(\mathrm{~s}, 3 \mathrm{H}), 1.42-1.25(\mathrm{~m}, 2 \mathrm{H}), 1.14$ $(\mathrm{d}, J=6.7,0.9 \mathrm{~Hz}, 3 \mathrm{H}), 1.02(\mathrm{~s}, 3 \mathrm{H}) .{ }^{13} \mathrm{C} \mathrm{NMR}\left(101 \mathrm{MHz}, \mathrm{CD}_{3} \mathrm{COCD}_{3}\right) \delta 187.79(\mathrm{C}), 170.28(\mathrm{C}), 149.29$ (C), $147.31(\mathrm{C}), 124.13(\mathrm{CH}), 116.87\left(\mathrm{CH}_{2}\right), 108.59\left(\mathrm{CH}_{2}\right), 46.01(\mathrm{CH}), 43.75\left(\mathrm{CH}_{2}\right), 40.95(\mathrm{C}), 40.25(\mathrm{CH})$, $32.57\left(\mathrm{CH}_{2}\right), 31.31\left(\mathrm{CH}_{2}\right), 20.08\left(\mathrm{CH}_{3}\right), 17.74\left(\mathrm{CH}_{3}\right), 9.70\left(\mathrm{CH}_{3}\right)$. HRMS TOF $(\mathrm{ESI}+) \mathrm{m} / z$ calculated for $\mathrm{C}_{16} \mathrm{H}_{23} \mathrm{O}[\mathrm{M}+\mathrm{H}]^{+}$231.1749, found 231.1739.

\subsubsection{Synthesis of Compounds 21 and 22 (See Scheme 7)}

To solution of $8(290 \mathrm{mg}, 1.05 \mathrm{mmol})$ in dry toluene $(5 \mathrm{~mL})$ cooled at $-78^{\circ} \mathrm{C}, 1.5 \mathrm{~mL}$ of collidine was added under argon atmosphere and the resulting mixture was stirred for $15 \mathrm{~min}$ at room temperature, then $0.47 \mathrm{~mL}$ of TMSOTf $(2.5 \mathrm{mmol})$ were added and stirred for another $10 \mathrm{~min}$. The reaction mixture was diluted in hexane $(20 \mathrm{~mL})$ and was washed with brine $(3 \times 15 \mathrm{~mL})$, dried with anhydrous $\mathrm{Na}_{2} \mathrm{SO}_{4}$, and concentrated in vacuo. The crude mixture was dissolved in dry THF (10 mL), and then $5 \mathrm{~mL}$ of formaldehyde (37\% water solution) and $1 \mathrm{~g}$ of $\mathrm{Yb}(\mathrm{OTf})_{3}(1.6 \mathrm{mmol})$ were added under argon atmosphere and stirred for $24 \mathrm{~h}$ at room temperature. Upon consumption of the starting material, the reaction mixture was diluted with $60 \mathrm{~mL}$ of saturated $\mathrm{NaHCO}_{3}$ solution and then was extracted with EtOAc $(3 \times 35 \mathrm{~mL})$. The combined organic layers were washed with $1 \mathrm{~N} \mathrm{HCl}(3 \times 20 \mathrm{~mL})$, and brine $(3$ $\times 20 \mathrm{~mL}$ ), dried with anhydrous $\mathrm{Na}_{2} \mathrm{SO}_{4}$, and concentrated in vacuo. The crude product was purified by flash chromatography (H:MTBE, 1:3) to obtain $187 \mathrm{mg}$ of $8(64.5 \%) 62 \mathrm{mg}$ of $21(19.3 \%)$ and $22 \mathrm{mg}$ of $22(6.9 \%)$.

Compound 21. $[\alpha]_{\mathrm{D}}=+111.2(c=1, \mathrm{DCM}) .{ }^{1} \mathrm{H} \mathrm{NMR}\left(400 \mathrm{MHz}, \mathrm{CDCl}_{3}\right) \delta 5.79(\mathrm{~s}, 1 \mathrm{H}), 5.09(\mathrm{~s}, 1 \mathrm{H})$, $4.97(\mathrm{~s}, 1 \mathrm{H}), 4.60(\mathrm{~d}, J=13.6 \mathrm{~Hz}, 1 \mathrm{H}), 4.56(\mathrm{~d}, J=13.3 \mathrm{~Hz}, 1 \mathrm{H}), 4.06(\mathrm{dd}, J=11.4,3.1 \mathrm{~Hz}, 1 \mathrm{H}), 3.73(\mathrm{dd}, J$ $=11.4,6.6 \mathrm{~Hz}, 1 \mathrm{H}), 2.86(\mathrm{sa}, 1 \mathrm{H}), 2.52(\mathrm{dddd}, J=15.5,13.8,5.1,1.9 \mathrm{~Hz}, 1 \mathrm{H}), 2.43-2.28(\mathrm{~m}, 3 \mathrm{H}), 2.10(\mathrm{~s}$, 3H), 2.09-2.05 (m, 1H), 2.02-1.89 (m, 2H), 1.37 (qd, $J=12.8,4.2 \mathrm{~Hz}, 1 \mathrm{H}), 1.15(\mathrm{~s}, 3 \mathrm{H}), 1.14-1.09(\mathrm{~m}, 1 \mathrm{H})$, $1.00(\mathrm{~d}, J=6.8 \mathrm{~Hz}, 3 \mathrm{H}) .{ }^{13} \mathrm{C}$ NMR $\left(126 \mathrm{MHz}, \mathrm{CDCl}_{3}\right) \delta=202.27(\mathrm{C}), 170.66(\mathrm{C}), 170.05(\mathrm{C}), 147.21(\mathrm{C})$, $124.35(\mathrm{CH}), 112.03\left(\mathrm{CH}_{2}\right), 66.03\left(\mathrm{CH}_{2}\right), 60.25\left(\mathrm{CH}_{2}\right), 49.19(\mathrm{CH}), 44.27\left(\mathrm{CH}_{2}\right), 41.16(\mathrm{CH}), 39.83(\mathrm{C})$, $36.29(\mathrm{CH}), 32.97\left(\mathrm{CH}_{2}\right), 31.77\left(\mathrm{CH}_{2}\right), 20.96\left(\mathrm{CH}_{3}\right), 17.14\left(\mathrm{CH}_{3}\right), 12.12\left(\mathrm{CH}_{3}\right)$. HRMS TOF $(\mathrm{ESI}+) \mathrm{m} / z$ calculated for $\mathrm{C}_{18} \mathrm{H}_{27} \mathrm{O}_{4}[\mathrm{M}+\mathrm{H}]^{+} 307.1909$, found 307.1950.

Compound 22. $[\alpha]_{\mathrm{D}}=+18.1(c=1, \mathrm{DCM}) .{ }^{1} \mathrm{H}$ NMR $\left(400 \mathrm{MHz}, \mathrm{CDCl}_{3}\right) \delta 5.84(\mathrm{~s}, 1 \mathrm{H}), 5.11(\mathrm{~s}, 1 \mathrm{H})$, $4.99(\mathrm{~s}, 1 \mathrm{H}), 4.63-4.55(\mathrm{~m}, 2 \mathrm{H}), 3.90(\mathrm{dd}, J=10.8,9.6 \mathrm{~Hz}, 1 \mathrm{H}), 3.72(\mathrm{dd}, J=10.8,3.9 \mathrm{~Hz}, 1 \mathrm{H}), 2.69-2.60$ $(\mathrm{m}, 1 \mathrm{H}), 2.56-2.47(\mathrm{~m}, 2 \mathrm{H}), 2.36(\mathrm{ddd}, J=13.5,4.3,2.7 \mathrm{~Hz}, 1 \mathrm{H}), 2.11(\mathrm{~s}, 3 \mathrm{H}), 2.10-2.02(\mathrm{~m}, 2 \mathrm{H}), 1.89(\mathrm{dt}, J$ $=13.0,3.1 \mathrm{~Hz}, 1 \mathrm{H}), 1.45(\mathrm{t}, J=12.6 \mathrm{~Hz}, 1 \mathrm{H}), 1.43-1.32(\mathrm{~m}, 1 \mathrm{H}), 1.15(\mathrm{~s}, 3 \mathrm{H}), 1.00(\mathrm{~d}, J=7.1 \mathrm{~Hz}, 3 \mathrm{H})$. ${ }^{13} \mathrm{C}$ NMR $\left(126 \mathrm{MHz}_{\mathrm{CDCl}}\right) \delta 202.64(\mathrm{C}), 170.67(\mathrm{C}), 170.05(\mathrm{C}), 146.85(\mathrm{C}), 122.97(\mathrm{CH}), 112.15\left(\mathrm{CH}_{2}\right)$, $66.06\left(\mathrm{CH}_{2}\right), 61.07\left(\mathrm{CH}_{2}\right), 52.39(\mathrm{CH}), 44.42\left(\mathrm{CH}_{2}\right), 41.58(\mathrm{CH}), 39.97(\mathrm{C}), 36.15(\mathrm{CH}), 33.36\left(\mathrm{CH}_{2}\right), 33.02$ 
$\left(\mathrm{CH}_{2}\right), 21.01\left(\mathrm{CH}_{3}\right), 20.96\left(\mathrm{CH}_{3}\right), 11.47\left(\mathrm{CH}_{3}\right)$. HRMS TOF (ESI+) $\mathrm{m} / z$ calculated for $\mathrm{C}_{18} \mathrm{H}_{27} \mathrm{O}_{4}[\mathrm{M}+\mathrm{H}]^{+}$ 307.1909, found 307.1925.

\subsubsection{Synthesis of Compound 23 (See Scheme 7)}

A solution of 21 ( $20 \mathrm{mg}, 0.06 \mathrm{mmol})$ was stirred in $0.5 \mathrm{~mL}$ deuterated chloroform ( $\mathrm{HCl}: 0.01 \%)$ for 7 days at $4{ }^{\circ} \mathrm{C}$. Then, the solvent was evaporated, and the crude mixture was purified by flash chromatography (H:MTBE, 1:3) to obtain $11 \mathrm{mg}$ of $\mathbf{2 3}(66.7 \%)$ and $6 \mathrm{mg}$ of $\mathbf{2 1}(30 \%)$.

Compound 23: $[\alpha]_{\mathrm{D}}=+99.8(\mathrm{c}=1, \mathrm{DCM}) .{ }^{1} \mathrm{H} \mathrm{NMR}\left(600 \mathrm{MHz}, \mathrm{CDCl}_{3}\right) \delta 6.07(\mathrm{~s}, 1 \mathrm{H}), 5.89(\mathrm{~s}, 1 \mathrm{H})$, $5.25(\mathrm{~s}, 1 \mathrm{H}), 5.10(\mathrm{~s}, 1 \mathrm{H}), 4.99(\mathrm{~s}, 1 \mathrm{H}), 4.60(\mathrm{~d}, J=13.5 \mathrm{~Hz}, 1 \mathrm{H}), 4.57(\mathrm{~d}, J=13.3 \mathrm{~Hz}, 1 \mathrm{H}), 2.62-2.7(\mathrm{~m}$, $1 \mathrm{H}), 2.51-2.44(\mathrm{~m}, 2 \mathrm{H}), 2.37-2.31(\mathrm{~m}, 1 \mathrm{H}), 2.10(\mathrm{~s}, 3 \mathrm{H}), 2.03(\mathrm{dt}, J=13.1,2.8 \mathrm{~Hz}, 1 \mathrm{H}), 1.99-1.93(\mathrm{~m}$, $1 \mathrm{H}), 1.40(\mathrm{qd}, J=12.8,4.6 \mathrm{~Hz}, 1 \mathrm{H}), 1.25(\mathrm{t}, J=12.9 \mathrm{~Hz}, 1 \mathrm{H}), 1.12(\mathrm{~d}, J=6.8 \mathrm{~Hz}, 3 \mathrm{H}), 0.99(\mathrm{~s}, 3 \mathrm{H}) .{ }^{13} \mathrm{C}$ NMR $\left(151 \mathrm{MHz}, \mathrm{CDCl}_{3}\right) \delta 189.41$ (C), 170.68 (C), 170.20 (C), 147.34 (C), 146.45 (C), $124.81(\mathrm{CH}), 118.74$ $\left(\mathrm{CH}_{2}\right), 111.93\left(\mathrm{CH}_{2}\right), 66.02\left(\mathrm{CH}_{2}\right), 45.96(\mathrm{CH}), 44.05\left(\mathrm{CH}_{2}\right), 41.19(\mathrm{C}), 36.53(\mathrm{CH}), 32.99\left(\mathrm{CH}_{2}\right), 29.67$ $\left(\mathrm{CH}_{2}\right), 20.95\left(\mathrm{CH}_{3}\right), 18.24\left(\mathrm{CH}_{3}\right), 10.32\left(\mathrm{CH}_{3}\right)$. HRMS TOF (ESI+) $\mathrm{m} / z$ calculated for $\mathrm{C}_{18} \mathrm{H}_{25} \mathrm{O}_{3}[\mathrm{M}+\mathrm{H}]^{+}$ 289.1804, found 289.1836 .

\subsection{Antifeedant Activity}

Insect colonies maintained at ICA-CSIC were used for conducting the bioassays. M. persicae and R. padi were reared on bell pepper (Capsicum annuum) and Hordeum vulgare plants, respectively, and maintained at $22 \pm 1{ }^{\circ} \mathrm{C}$ and $>70 \%$ relative humidity, with a photoperiod of $16: 8 \mathrm{~h}$ (L:D) in a growth chamber.

Antifeedant bioassays: The upper surface of $C$. anuum and H. vulgare leaf disks or fragments $\left(1.0 \mathrm{~cm}^{2}\right)$ were treated with $10 \mu \mathrm{l}$ of the test substance. The products were tested at an initial dose of $5 \mathrm{mg} / \mathrm{ml}\left(50 \mu \mathrm{g} / \mathrm{cm}^{2}\right)$ respectively. Twenty ventilated plastic boxes $(2 \times 2 \mathrm{~cm})$ with 10 apterous aphid adults (24-48 h old) each were allowed to feed in a growth chamber $(24 \mathrm{~h}$, environmental conditions as above). Each experiment was repeated $2(\mathrm{SE}<10 \%)$ and terminated after $24 \mathrm{~h}$. Aphid settling was measured by counting the number of aphids on each leaf fragment. Settling inhibition (\%FI or $\% \mathrm{SI})$ was calculated as $\% \mathrm{FI} / \% \mathrm{SI}=[1-(\mathrm{T} / \mathrm{C}) \times 100]$, where $\mathrm{T}$ and $\mathrm{C}$ represent settling on treated and control leaf disks, respectively. The antifeedant effects (\% SI) were analyzed for significance by the nonparametric Wilcoxon signed-rank test. Extracts and compounds with an SI $>70 \%$ were further tested in a dose-response experiment (3-4 serial dilutions) to calculate their relative potency (EC50, the effective dose to give a 50\% settling reduction) from linear regression analysis (\% FI/SI on Log-dose) [27].

\subsection{Ixodicidal Activity}

H. lusitanicum engorged female ticks were collected in central Spain (Finca La Garganta, Ciudad Real) from their host (deer) and maintained at $22-24{ }^{\circ} \mathrm{C}$ and $70 \% \mathrm{RH}$ until oviposition and egg hatching. Resulting larvae (4-6 weeks old) were used for the bioassays [12]. Briefly, $50 \mu \mathrm{L}$ of test solution were added to $25 \mathrm{mg}$ of powdered cellulose at different concentrations (initial concentration of $10 \mathrm{mg} / \mathrm{ml}$ ) and the solvent was evaporated. For each test, three replicates with 20 larvae each were used. Dead ticks were counted after $24 \mathrm{~h}$ of contact with the treated cellulose at the environmental conditions described, using a binocular magnifying glass. The larvicidal activity data are presented as percent mortality corrected according to Schneider-Orelli's formula. Effective lethal doses $\left(\mathrm{LC}_{50}\right.$ and $\mathrm{LC}_{90}$ ) were calculated by Probit Analysis (5 serial dilutions, STATGRAPHICS Centurion XVI, version 16.1.02, Statgraphics Technologies, Inc., P.O. Box 134, The Plains, Virginia 20198, USA). 


\section{Results and Discussion}

\subsection{Synthesis of Nootkatone Derivatives}

The generation of chemical diversity from nootkatone (1) started by subjecting this molecule to the iodine/DMSO dehydrogenating system [28,29]. The reaction of 1 with 0.1 equiv of $I_{2}$ and 5 equiv of DMSO in refluxing toluene led to the production of three dehydrogenated products $\mathbf{2 - 4}$ (Table 1 , entry 1). Under these conditions, dienone 3 was obtained as the major compound [23]. Compound $\mathbf{2}$ is a natural component of grapefruit juice [22]. Compound 4 is described for the first time here. Its stereochemistry at C3 was assigned based on the proton $J$ value $(J=12.5 \mathrm{~Hz})$ of the proton at this position.

Table 1. Reaction of nootkatone with the dehydrogenating system $\mathrm{I}_{2} / \mathrm{DMSO}$.

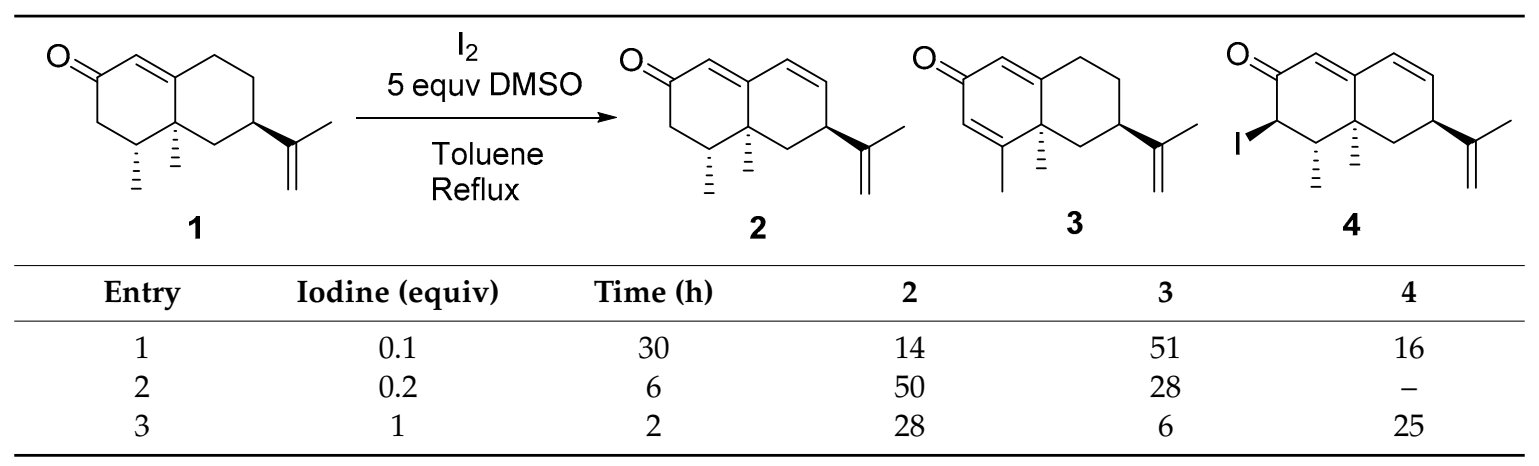

When $0.2 \mathrm{mmol}$ of $\mathrm{I}_{2}$ were used (Table 1, entry 2), the same mixture of compounds was obtained, with compound 2 being the main reaction product ( $50 \%$ yield). When the quantity of $\mathrm{I}_{2}$ increased to $1 \mathrm{mmol}$ (Table 1, entry 3), a different proportion of the same mixture $\mathbf{2} \mathbf{- 4}$ was obtained, with compounds $2(28 \%)$ and $4(25 \%)$ being then the main reaction products. A mechanistic proposal for the formation of these substances is shown in Scheme 1. Thus, when $\mathbf{1}$ reacts via Ia, compounds $\mathbf{2}$ and $\mathbf{4}$ are obtained, whereas if $\mathbf{I b}$ is the reacting species, the process leads to the generation of $\mathbf{3}$.

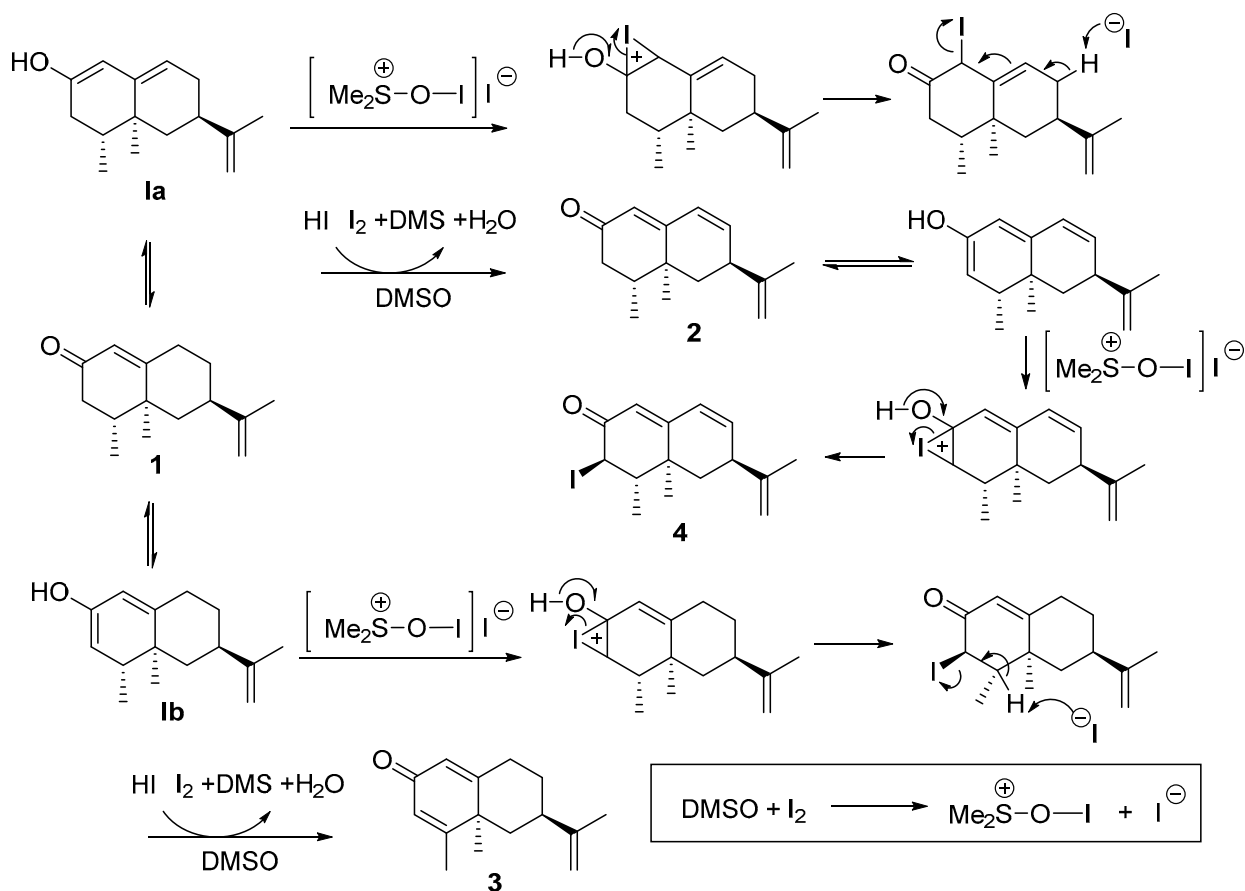

Scheme 1. Proposed mechanism for the generation of $2-4$. 
The regioselective dehydrogenation of nootkatone also afforded dienone $3(62 \%)$ after its treatment with $\mathrm{DDQ} / \mathrm{AcOH}$ in benzene.

The oxidation of 1 with $\mathrm{SeO}_{2}$ in refluxing EtOH for $3 \mathrm{~h}$ led to compounds 5-7 (Scheme 2), with alcohol 5 being the major product. Compound 7 was formed after allylic oxidation at $\mathrm{C7}$ and subsequent Michael addition of this hydroxyl group to C10. Alcohol 5 is a natural product isolated from Alpinia oxyphylla [30], and it was also found in P450-catalyzed transformations of nootkatone [31]. Compound $\mathbf{6}$ was previously synthesized from nootkatone by a photochemical process [24].
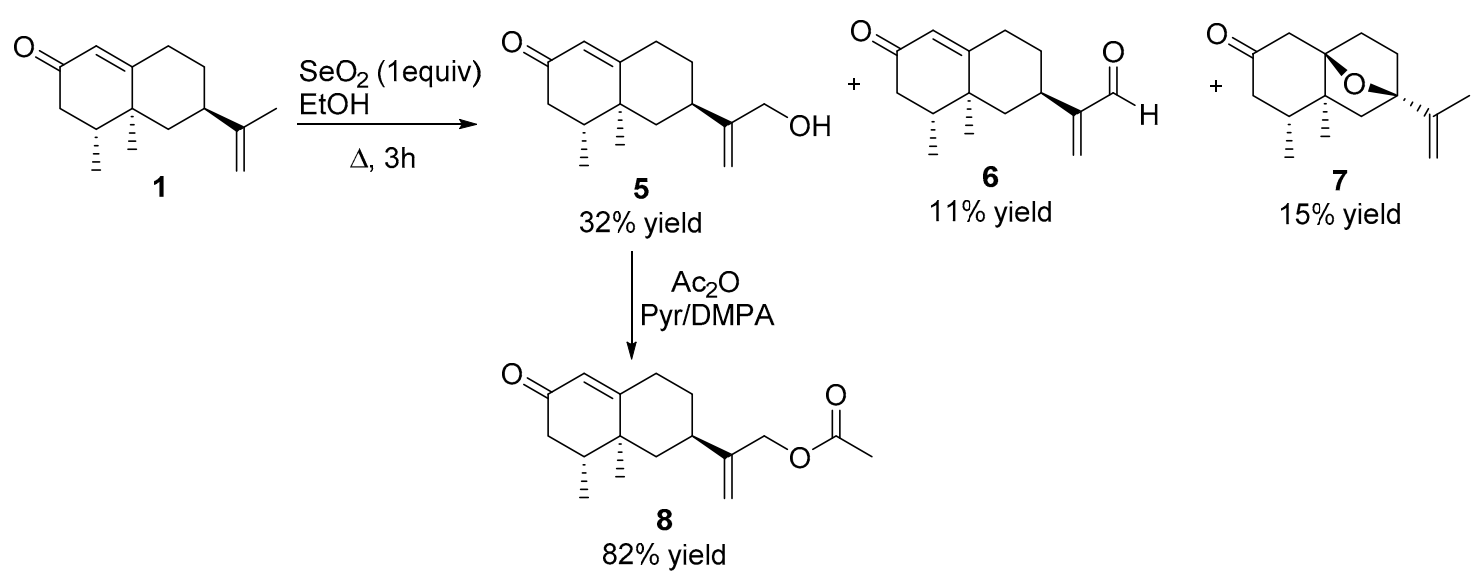

Scheme 2. Synthesis of nootkatone derivatives 5-8.

Due to the overlapping signals observed for compound 7 in its ${ }^{1} \mathrm{H}-\mathrm{NMR}$ spectrum, the configurations at C17 and C10 could only be only assigned after analysis of the corresponding stepNOESY experiments (selective TOCSY edited preparation NOESY experiences) [32] (Figure 2).

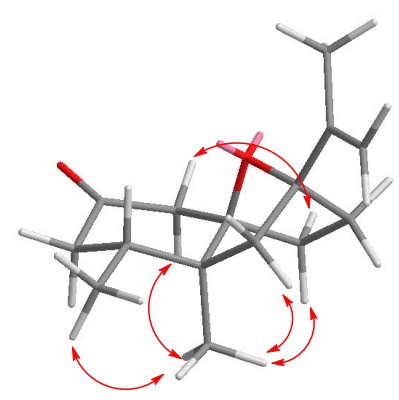

Figure 2. Selected NOEs observed for compound 7.

Oxidation of dienone $\mathbf{3}$ with $\mathrm{SeO}_{2}$ under the same experimental conditions gave 9-10 with acceptable yields (Scheme 3).

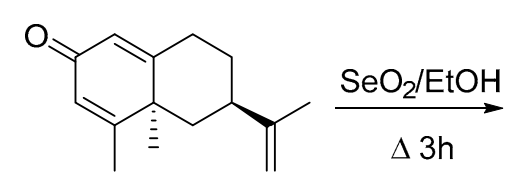

3<smiles>C=C(CO)[C@H]1CCC2=CC(=O)C=C(C)[C@]2(C)C1</smiles>

9

$53 \%$ yield<smiles>C=C(C=O)C1CCC2=CC(=O)C=C(C)[C@H]2C1</smiles>

10

$14 \%$ yield

Scheme 3. Synthesis of nootkatone derivatives 9-10.

Epoxidation of $\mathbf{1}$ with $m \mathrm{CPBA}$ at $0{ }^{\circ} \mathrm{C}$ for $3 \mathrm{~h}$ afforded mainly monoepoxide 11 [33]. With longer reaction times reaction times, excess of $m \mathrm{CPBA}$ and at room temperature, the mixture of epoxides 11 
and 12 [26] was obtained along with diepoxide 13, generated via the Baeyer-Villiger oxidation of the C1-C2 bond of diepoxide 12 (Scheme 4).<smiles>C=C(C)C1CCC2=CC(=O)C[C@H](C)[C@@]2(C)C1</smiles>

Scheme 4. Synthesis of nootkatone derivatives 11-12.

Then the reactivity of the $\alpha$-carbonyl methylene of nootkatone to trigger either oxidations or carbon homologations was addressed. The incorporation of a hydroxyl group at $\mathrm{C} 3$ was achieved via treatment of 1 with TMSOTf (trimethylsilyl trifluoromethanesulfonate) and collidine to produce the TMS-dienol intermediate I, which after a Rubottom oxidation [34] evolved towards the corresponding $\alpha$-hydroxy carbonyl stereoisomers 14-15. These compounds were easily oxidized with the Dess Martin periodane to afford the dicarbonyl derivatives 16-17 (Scheme 5).

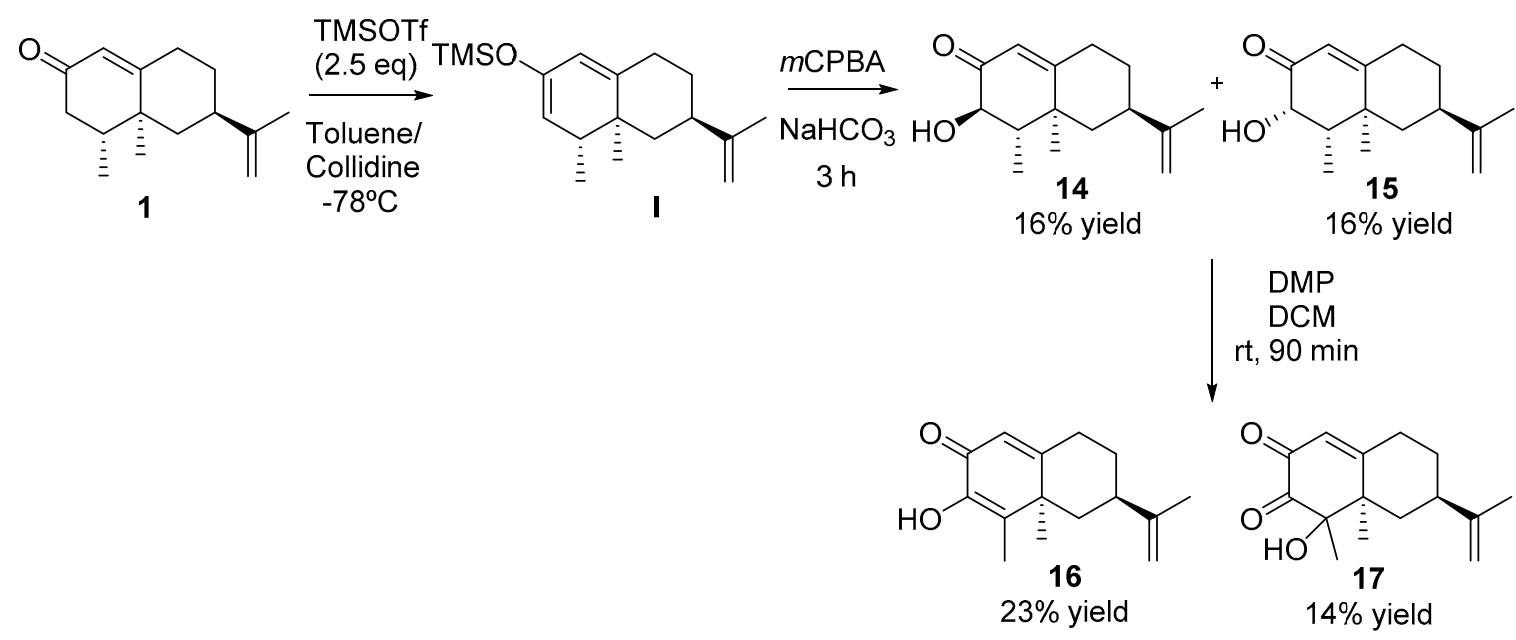

Scheme 5. Synthesis of nootkatone derivatives 14-17.

As happened with 4 , the stereochemistry at $\mathrm{C} 3$ of alcohols 14 and 15 was determined on the basis of the coupling constant values measured for $\mathrm{H} 3(14: \mathrm{H} 3, \mathrm{~d}, J=12.7 ; 15$ : $\mathrm{H} 3, \mathrm{~d} J=5.2)$ : Additionally, the correlations observed in the 2D NOESY spectrum of 14 confirmed this assignment (Figure 3 ).

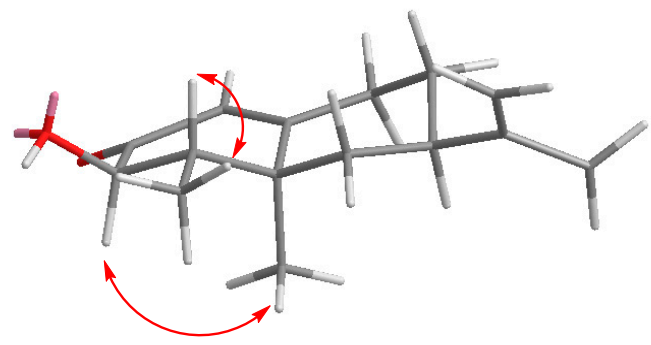

Figure 3. Selected NOESY correlations observed for compound $\mathbf{1 4}$.

Additionally, the same TMS-dienol I was condensated with formaldehyde (37\% in water) using $\mathrm{Yb}(\mathrm{OTf})_{3}$ as catalyst [35]. Under these conditions aldol 18 was obtained in a 34\% yield together with minor proportions of compound $19(11 \%)$, generated a result of a double condensation process. 
Starting from 18, the elimination of the hydroxyl group was achieved in two steps following the Grieco protocol [36] to obtain a good yield of dienone 20 in good (71\%) (Scheme 6).<smiles>C=C(C)[C@@H]1CCC2=CC(OC)=C[C@H](C)[C@]2(C)C1</smiles>

I
$\mathrm{HCOH}$

$\stackrel{37 \% \text { in water }}{\longrightarrow}$

$\mathrm{Yb}(\mathrm{OTf})_{3}$

$\mathrm{rt}, 24 \mathrm{~h}$<smiles>C=C(C)C1CCC2=CC(=O)[C@H](CO)[C@H](C)[C@]2(C)C1</smiles>

18<smiles>C=C(C)[C@@H]1CCC2=CC(=O)C(C)(CO)[C@H](C)[C@H]2C1</smiles>

19

$34 \%$ yield

$11 \%$ yield

a) $\mathrm{o}-\mathrm{NO}_{2} \mathrm{PhSeCN}$ (1.5 equiv) $n-\mathrm{Bu}_{3} \mathrm{P}$ ( 1.5 equiv)

THF, rt, 80 min

b) $\mathrm{H}_{2} \mathrm{O}_{2}(30 \%)$

THF,rt, 15 min

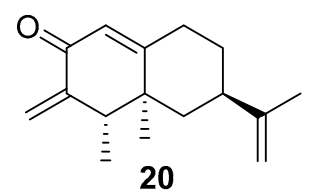

$71 \%$ yield

Scheme 6. Synthesis of nootkatone derivatives 18-20.

The C3 stereochemistry of alcohol $\mathbf{1 8}$ was determined following analysis of the NOEs observed in the selective 1D-NOESY spectra of this compound (Figure 4).

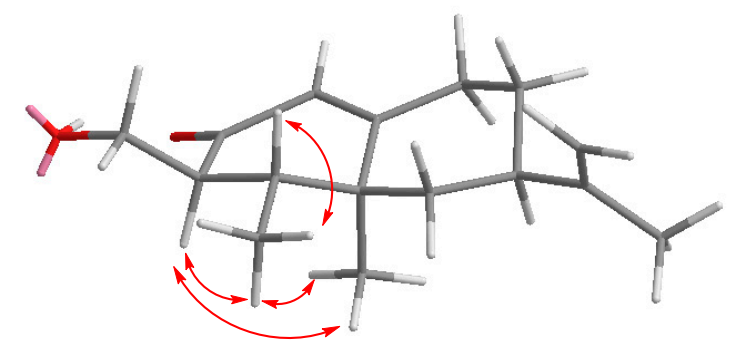

Figure 4. Selected NOEs observed for compound 18.

Following a similar sequence, the acetoxy derivative $\mathbf{2 3}$ was synthesized from $\mathbf{8}$ in three steps (Scheme 7).<smiles>C=C(COC(C)=O)C1CCC2=CC(OC)=C[C@H](C)[C@H]2C1</smiles>

8 $-78^{\circ} \mathrm{C}$

$\mathrm{HCOH}$ ( $37 \%$ in water) $\mathrm{Yb}(\mathrm{OTf})_{3}(0.9$ equiv)<smiles>C=C(COC(C)=O)C1CCC2=CC(=O)C(=C)[C@]2(C)C1</smiles>

$24 \%$ yield three steps<smiles>ClC(I)(I)I</smiles>
$\checkmark \quad r t, 24 \mathrm{~h}$<smiles>C=C(COC(C)=O)[C@@H]1CCC2=CC(=O)[C@H](CO)[C@H](C)[C@H]2C1</smiles>

21: $3 S$

22: $3 R$

Scheme 7. Synthesis of nootkatone derivatives 21-23. 
The enhancements observed in the selective 1D NOESY of 21 allowed us to assign the stereochemistry of this compound at C3 (Figure 5).

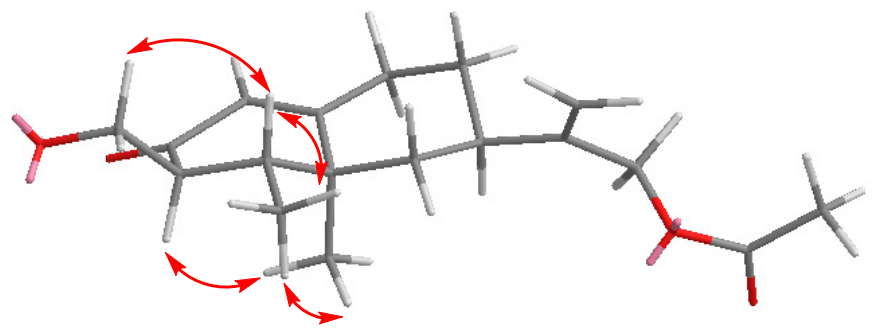

Figure 5. Selected NOEs observed for compound 21.

\subsection{Antifeedant Activity}

Table 2 shows the results of the aphid antifeedant effects of nootkatone (1) and derivatives. These compounds were also tested against the lepidopteran $S$. littoralis without significant effects (all $\mathrm{EC}_{50}>50$ ). The most active compounds were 20 (both species), 15 and 2 (R. padi) followed by 7 , 10 (M. persicae), 3 (R. padi) and $\mathbf{1 4}$ (both species). All these derivatives were more active than nootkatone (1), with 20, 15 and 2 being over 50\% more potent, followed by 10, 7, and $\mathbf{1 1}(\sim 50 \%)$ (Figure 6 ).

It should be noticed that the $\alpha, \beta$-unsaturated ketone 20, with an extra methylene, 15 with an $\alpha$-hydroxy at C-3 and 2, with an additional endocyclic C8-C9 unsaturation, exhibited strong antifeedant effects followed by the exocyclic epoxide 7 , and the aldehyde derivative 10 . The presence of an additional Michael acceptor in compound $\mathbf{2 0}$ may account for the observed bioactivity of this product. In this regard, previous results showed that the presence of an exocyclic epoxide in eremophilanes resulted in strong antifeedant effects against M. persicae and S. littoralis [12].

Table 2. Insect antifeedant effects of nootkatone (1) and derivatives 2-23 against aphids.

\begin{tabular}{ccccc}
\hline \multirow{2}{*}{ Product } & \multicolumn{2}{c}{ M. persicae } & \multicolumn{2}{c}{ R. padi } \\
\cline { 2 - 5 } & $\mathbf{5 0}\left(\boldsymbol{\mu g} / \mathbf{c m}^{\mathbf{2}}\right)^{\mathbf{a}}$ & $\mathbf{E C}_{\mathbf{5 0}} \mathbf{b}$ & $\mathbf{5 0}\left(\boldsymbol{\mu g} / \mathbf{c m}^{\mathbf{2}}\right)^{\mathbf{a}}$ & $\mathbf{E C}_{\mathbf{5 0}} \mathbf{b}$ \\
\hline $\mathbf{1}$ & $50.7 \pm 9.1$ & $\sim 50$ & $57.7 \pm 10.2$ & $\sim 50$ \\
$\mathbf{2}$ & $35.4 \pm 9.0$ & $>50$ & $86.0 \pm 3.6^{*}$ & $17.4(14.4-20.9)$ \\
$\mathbf{3}$ & $24.6 \pm 7.5$ & $>50$ & $77.1 \pm 4.5^{*}$ & $27.1(20.7-35.4)$ \\
$\mathbf{5}$ & $49.4 \pm 10.9$ & $\sim 50$ & $40.9 \pm 8.2$ & $>50$ \\
$\mathbf{6}$ & $57.2 \pm 10.1$ & $\sim 50$ & $30.7 \pm 6.8$ & $>50$ \\
$\mathbf{7}$ & $79.9 \pm 5.4^{*}$ & $\sim 25$ & $30.8 \pm 6.5$ & $>50$ \\
$\mathbf{8}$ & $37.8 \pm 9.8$ & $>50$ & $31.4 \pm 5.9$ & $>50$ \\
$\mathbf{9}$ & $34.2 \pm 8.5$ & $>50$ & $44.2 \pm 9.2$ & $>50$ \\
$\mathbf{1 0}$ & $80.6 \pm 4.8^{*}$ & $23.5(16.6-33.3)$ & $36.95 \pm 8.5$ & $>50$ \\
$\mathbf{1 1}$ & $49.0 \pm 9.4$ & $\sim 50$ & $69.4 \pm 7.6^{*}$ & $\sim 25$ \\
$\mathbf{1 2}$ & $59.1 \pm 7.0$ & $\sim 50$ & $51.8 \pm 5.7$ & $\sim 50$ \\
$\mathbf{1 3}$ & $29.0 \pm 4.5$ & $>50$ & $19.85 \pm 6.5$ & $>50$ \\
$\mathbf{1 4}$ & $91.2 \pm 5.3^{*}$ & $30-40$ & $83.8 \pm 4.9 *$ & $30-40>50$ \\
$\mathbf{1 5}$ & $54.5 \pm 8.0$ & $\sim 50$ & $78.81 \pm 4.4$ & $8.3(5.5-12.7)$ \\
$\mathbf{1 6}$ & $63.9 \pm 7.4^{*}$ & $\sim 50$ & $37.3 \pm 7.3$ & $>50$ \\
$\mathbf{1 7}$ & $40.6 \pm 6.9$ & $>50$ & $34.2 \pm 7.9$ & $>50$ \\
$\mathbf{1 8}$ & $60.4 \pm 7.1^{*}$ & $\sim 50$ & $44.4 \pm 9.0$ & $>50$ \\
$\mathbf{1 9}$ & $35.4 \pm 7.6$ & $>50$ & $19.8 \pm 5.6$ & $>50$ \\
$\mathbf{2 0}$ & $92.1 \pm 2.5^{* *}$ & $16.3(13.6-19.7)$ & $93.9 \pm 4.2 *$ & $7.1(5.4-9.4)$ \\
$\mathbf{2 3}$ & $62.3 \pm 9.2$ & $\sim 50$ & $17.5 \pm 6.7$ & $>50$ \\
\hline
\end{tabular}

${ }^{a}$ Percent settling inhibition (\%SI, $n=100$ insects). ${ }^{b}$ Dose needed to give $50 \%$ settling inhibition ( $95 \%$ Confidence Limits). * Significantly different from the control $(p<0.05)$, Wilcoxon paired rank test. 


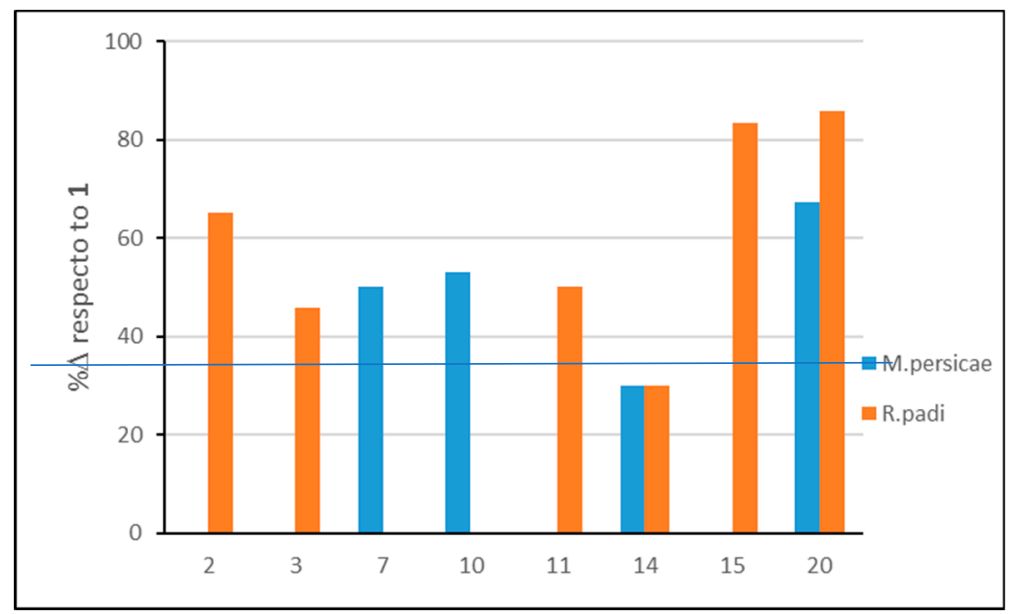

Figure 6. Increment $(\% \Delta)$ in antifeedant activity $\left(\mathrm{EC}_{50}\right.$ values $)$ of derivatives $2,3, \mathbf{7}, \mathbf{1 0}, \mathbf{1 1}, \mathbf{1 4}, \mathbf{1 5}$, and 20 with respect to 1 .

\subsection{Ixodicidal Activity}

Table 3 shows the ixodicidal effects of nootkatone (1) and derivatives against H. lusitanicum larvae.

Table 3. Acaricidal activity of nootkatone (1) and derivatives 2-23 against H. lusitanicum larvae.

\begin{tabular}{|c|c|c|c|}
\hline \multirow{2}{*}{ Product } & \multirow{2}{*}{$\%$ Mortality ${ }^{a}(20 \mu \mathrm{g} / \mathrm{mg})$} & \multicolumn{2}{|c|}{ 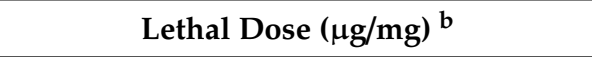 } \\
\hline & & $\mathrm{LD}_{50}$ & $\mathrm{LD}_{90}$ \\
\hline 1 & $85.9 \pm 2.2$ & $4.02(1.92-7.42)$ & $18.02(13.60-29.16)$ \\
\hline 2 & $100 \pm 0$ & $5.36(4.94-5.90)$ & $4.31(3.93-4.82)$ \\
\hline 3 & $98.3 \pm 0.4$ & $2.94(2.54-3.38)$ & $5.72(5.0-6.80)$ \\
\hline 5 & $100 \pm 0$ & $0.96(0.88-1.04)$ & $1.28(1.18-1.38)$ \\
\hline 6 & $47.3 \pm 14.3$ & & \\
\hline 7 & $100 \pm 0$ & $5.04(4.56-5.64)$ & $8.04(7.20-9.22)$ \\
\hline 8 & $32.2 \pm 3.0$ & & \\
\hline 9 & $42.6 \pm 2.2$ & & \\
\hline 10 & $8.5 \pm 1.0$ & & \\
\hline 11 & $88.5 \pm 1.6$ & $5.60(4.88-6.44)$ & $10.84(9.50-12.8)$ \\
\hline 12 & $1.5 \pm 1.6$ & & \\
\hline 13 & $7.7 \pm 10.8$ & & \\
\hline 14 & $46.3 \pm 8.7$ & & \\
\hline 15 & $100 \pm 0$ & $8.74(8.10-9.40)$ & $11.52(10.68-12.8)$ \\
\hline 16 & $23.4 \pm 3.5$ & & \\
\hline 17 & $11.7 \pm 7.3$ & & \\
\hline 18 & $96.7 \pm 1.6$ & $7.06(6.20-8.10)$ & $12.94(11.34-15.36)$ \\
\hline 19 & $17.7 \pm 1.3$ & & \\
\hline 20 & $100 \pm 0$ & $1.34(1.20-1.50)$ & $2.14(1.92-2.44)$ \\
\hline 23 & $11.5 \pm 1.3$ & & \\
\hline
\end{tabular}

a Mortality data corrected according to Schneider-Orelli's formula. ${ }^{\mathrm{b}}$ Doses needed to give 50 and $90 \%$ mortality (95\% Confidence Limits).

Using the activity of nootkatone as reference, the tested compounds can be classified in four groups according to their relative activity (Figure 7). Compounds 5, $20(>50 \%)$ and 3 (20 and 68\%) showed an increase in acaricidal activity with respect to nootkatone (1) for both lethal doses (Table 3 , Figure 5); followed by 2, 7 and 11 with an increase for $\mathrm{LD}_{90}>50 \%$. Compounds 15 and 18 showed reduced acaricidal potency at $\mathrm{LD}_{50}$ and higher at $\mathrm{LD}_{90}$ than 1 . Lastly, the remaining compounds either displayed a significant loss of activity or were not active at all. These results are relevant considering 
that nootkatone was reported to be more active against the tick species Ixodes scapularis and Amblyomma americanum than Permanone, a permethrin-based commercial clothing repellent [37].

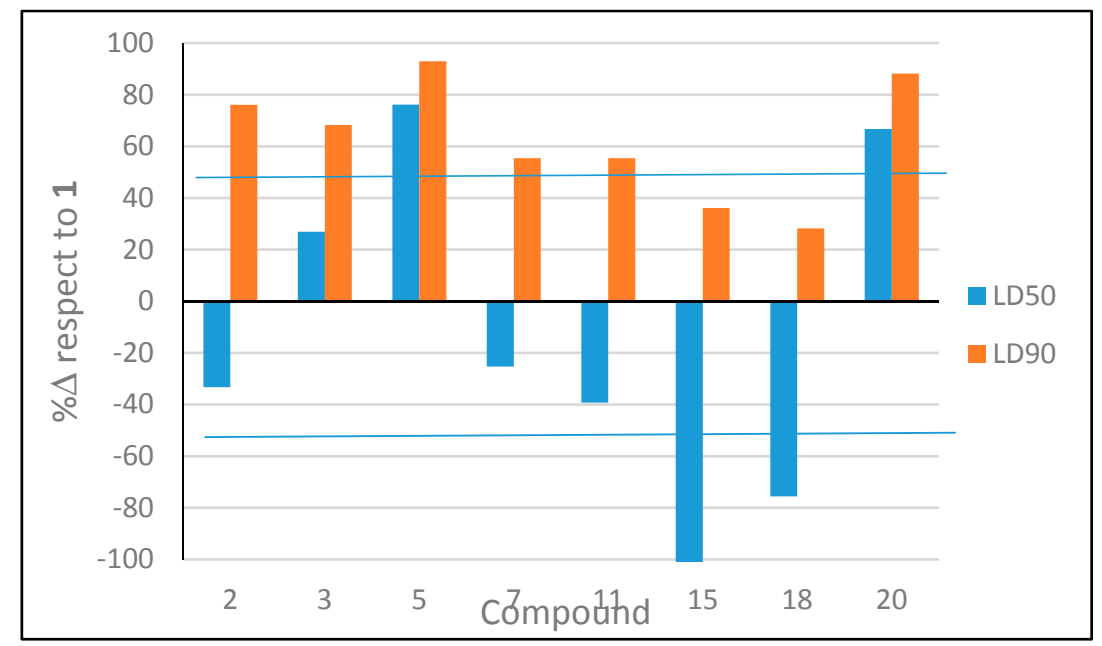

Figure 7. Increment $(\% \Delta)$ in ixodicidal activity $\left(\mathrm{LD}_{50}\right.$ and $\mathrm{LD}_{90}$ values) of derivatives $\mathbf{2 , 3}, \mathbf{5}, \mathbf{7}, \mathbf{1 1}, \mathbf{1 5}$, 18, and 20 respect to 1 .

From these data, the following structure-activity relationships were inferred for the acaricidal effects. Hydroxylation at C13 (compound 5) increased acaricidal activity, possibly due to a decrease in the volatility of the molecule. The presence of a methylene group at C3 (compound 20) also increased activity with respect to nootkatone, most likely due to the presence of a more accessible Michael acceptor. Also, an additional endocyclic unsaturation increased the ixodicidal effects (C3-C4 in 3 and C8-C9 in 2). Finally, it can be concluded that the unsaturated ketone present in the structure of 1 plays a key role in the antitick activity of the tested substances whereas the C11-C12 double bond enhances the antitick efficiency as previously shown [12].

\section{Conclusions}

Starting from the natural product nootkatone (1), a known biopesticide compound, we have synthesized 22 derivatives in a reduced number of steps. Two are known natural products (2 and 5$)$ and 15 have been synthesized for the first time. With these derivatives, we performed antifeedant test against $S$. littoralis, $M$. persicae and $R$. padi, and ixodicidal tests against $H$. lusitanicum. Compounds 20, 14 and 2 were more antifeedant (>50\% more potent) than 1, followed by 7, 10 and $\mathbf{1 1}(\sim 50 \%)$. Compounds 5, 20, and $\mathbf{3}$ were stronger acaricidal agents than $\mathbf{1}$ for both lethal doses, followed by 2, 7 and 11 ( $\mathrm{LD}_{90}>50 \%$ ). Compound 20 was the only compound with stronger effects than 1 on both targets (insects and ticks). In this regard, the introduction of an additional methylene moiety at C3 enhanced antifeedant and ixodicidal activities. The significance of these results is supported by the fact that nootkatone is subject to research and development to support US Environmental Protection Agency registration for commercial marketing as a repellent and biopesticide against mosquitoes and ticks [38].

Supplementary Materials: The supplementary material related to this article includes NMR spectra of new compounds and is available online.

Author Contributions: Conceptualization, A.F.B. A.G.-C. and J.F.Q.d.M; Investigation, A.G.P., H.P.d.P., A.S.O. and A.G.-C.; Writing-Original Draft Preparation, A.F.B. and J.F.Q.d.M; Writing-Review \& Editing, A.F.B., A.G.-C. and J.F.Q.d.M; Funding Acquisition, A.F.B., A.G.-C. and J.F.Q.d.M.

Funding: This research was funded by Grant CTQ-2015-64049-C3-3-R / CTQ2015-64049-C3-1-R; Ministerio de Economía y Competitividad, Spain (MINECO/FEDER).

Conflicts of Interest: The authors declare no conflict of interest. 


\section{References}

1. Marrone, P.G. Pesticidal natural products - status and future potential. Pest Manag. Sci. 2019, 75, $2325-2340$. [CrossRef] [PubMed]

2. Cantrell, C.L.; Dayan, F.E.; Duke, S.O. Natural products as sources for new pesticides. J. Nat. Prod. 2012, 75, 1231-1242. [CrossRef] [PubMed]

3. Erdtman, H.; Hirose, Y. The chemistry of the natural order Cupressales. XLVI. The structure of nootkatone. Acta Chem. Scand. 1962, 16, 1311-1314. [CrossRef]

4. Karchesy, J.J.; Kelsey, R.G.; González-Hernández, M.P. Yellow-Cedar, Callitropsis (Chamaecyparis) nootkatensis, secondary metabolites, biological activities, and chemical ecology. J. Chem. Ecol. 2018, 44, 510-524. [CrossRef]

5. Kahrilas, G.A.; Wally, L.M.; Fredrick, S.J.; Hiskey, M.; Prieto, A.L.; Owens, J.E. Microwave-assisted green synthesis of silver nanoparticles using orange peel extract. ACS Sustain. Chem. Eng. 2014, 2, 367-376. [CrossRef]

6. Andersen, N.H. Biogenetic implications of the antipodal sesquiterpenes of vetiver oil. Phytochemistry 1970, 9, 145-151. [CrossRef]

7. Zorn, H.; Taupp, D.E.; Huelsdau, B.; Scheibner, M.; Fraatz, M.A.; Berger, R.G. “Bioflavors”-An excursion from the garlic mushroom to raspberry aroma. In Proceedings of the Recent Highlights Flavor Chemistry and Biology, Eisenach, Germany, 27 February-2 March 2007.

8. Panella, N.A.; Dolan, M.C.; Karchesy, J.J.; Xiong, Y.; Peralta-Cruz, J.; Khasawneh, M.; Montenieri, J.A.; Maupin, G.O. Use of novel compounds for pest control: insecticidal and acaricidal activity of essential oil components from heartwood of Alaska yellow cedar. J. Med. Entomol. 2005, 42, 352-358. [CrossRef]

9. Zhu, B.C.R.; Henderson, G.; Chen, F.; Maistrello, L.; Laine, R.A. Nootkatone is a repellent for formosan subterranean termite (Coptotermes formosanus). J. Chem. Ecol. 2001, 27, 523-531. [CrossRef]

10. Julien, B.N.; Dolan, M.C.; Bradbury, R.S. Nootkatone compositions and methods for repelling and killing ticks and detachment of feeding ticks. PCT Int. Appl. 2017. WO 2017162886 A1 20170928.

11. Addesso, K.M.; Oliver, J.B.; O’Neal, P.A.; Youssef, N. Efficacy of nootka oil as a biopesticide for management of imported fire ants (Hymenoptera: Formicidae). J. Econ. Entomol. 2017, 110, 1547-1555. [CrossRef]

12. Ruiz-Vásquez, L.; Olmeda, A.S.; Zúñiga, G.; Villarroel, L.; Echeverri, L.F.; González-Coloma, A.; Reina, M. Insect antifeedant and ixodicidal compounds from Senecio adenotrichius. Chem. Biodivers. 2017, 14, e1600155. [CrossRef] [PubMed]

13. Guo, Y.; Zhang, Q.; Liu, Z.; Bao, C.; Fan, J.; Yang, R. Non-food bioactive products: Design and semisynthesis of novel (+)-nootkatone derivatives containing isoxazoline moiety as insecticide candidates. Ind. Crops Prod. 2019, 140, 111706. [CrossRef]

14. Handore, K.L.; Kalmode, H.P.; Sayyad, S.; Seetharamsingh, B.; Gathalkar, G.; Padole, S.; Pawar, P.V.; Joseph, M.; Sen, A.; Reddy, D.S. Insect-repellent and mosquitocidal effects of noreremophilane- and nardoaristolone-based compounds. ACS Omega 2019, 4, 2188-2195. [CrossRef]

15. Bradbury, R.S.; Amick, J.D. Nootkatone insecticidal emulsion for repelling, knocking down, or killing pests. PCT Int. Appl. 2018. WO 2018100113 A1 20180607.

16. Anderson, J.A.; Coats, J.R. Acetylcholinesterase inhibition by nootkatone and carvacrol in arthropods. Pestic. Biochem. Physiol. 2012, 102, 124-128. [CrossRef]

17. Munoz, L.; Rosell, G.; Quero, C.; Guerrero, A. Biosynthetic pathways of the pheromone of the Egyptian armyworm Spodoptera littoralis. Physiol. Entomol. 2008, 33, 275-290. [CrossRef]

18. Kim, J.H.; Jander, G. Myzus persicae (green peach aphid) feeding on Arabidopsis induces the formation of a deterrent indole glucosinolate. Plant J. 2007, 49, 1008-1019. [CrossRef]

19. Greenslade, A.F.C.; Ward, J.L.; Martin, J.L.; Corol, D.I.; Clark, S.J.; Smart, L.E.; Aradottir, G.I. Triticum monococcum lines with distinct metabolic phenotypes and phloem-based partial resistance to the bird cherry-oat aphid Rhopalosiphum padi. Ann. Appl. Biol. 2016, 168, 435-449. [CrossRef]

20. ECDC. European Centre for disease prevention and control. Hyalomma marginatum. Available online: https: //ecdc.europa.eu/en/disease-vectors/facts/tick-factsheets/hyalomma-marginatum (accessed on 13 October 2019).

21. Galloway, W.R.J.D.; Isidro-Llobet, A.; Spring, D.R. Diversity-oriented synthesis as a tool for the discovery of novel biologically active small molecules. Nat. Commun. 2010, 1, 80. [CrossRef] 
22. Demole, E.; Enggist, P. Further investigation of grapefruit juice flavor components (Citrus paradisi MACFAYDEN). Valencane- and eudesmane-type sesquiterpene ketones. Helv. Chim. Acta 1983, 66, 1381-1391. [CrossRef]

23. Caine, D.; Chu, C.-Y.; Graham, S.L. Photochemical pathways for the interconversion of nootkatane and spirovetivane sesquiterpenes. J. Org. Chem. 1980, 45, 3790-3797. [CrossRef]

24. Tateba, H.; Morita, K.; Tada, M. Photochemical reaction of nootkatone under various conditions. J. Chem. Res. Synopses 1992, 140-141.

25. Furusawa, M.; Hashimoto, T.; Noma, Y.; Asakawa, Y. Biotransformation of citrus aromatics nootkatone and valencene by microorganisms. Chem. Pharm. Bull. 2005, 53, 1423-1429. [CrossRef] [PubMed]

26. Khasawneh, M.A.; Xiong, Y.; Peralta-Cruz, J.; Karchesy, J.J. Biologically important eremophilane sesquiterpenes from Alaska cedar keartwood essential oil and their semi-synthetic Derivatives. Molecules 2011, 16, 4775-4785. [CrossRef]

27. Santana, O.; Andrés, M.F.; Sanz, J.; Errahmani, N.; Abdeslam, L.; González-Coloma, A. Valorization of essential oils from Moroccan aromatic plants. Nat. Prod. Commun. 2014, 9, 1109-1114. [CrossRef]

28. Domingo, V.; Prieto, C.; Castillo, A.; Silva, L.; Quilez del Moral, J.F.; Barrero, A.F. Iodine-promoted metal-free aromatization: Synthesis of biaryls, oligo p-phenylenes and A-ring modified steroids. Adv. Synth. Catal. 2015, 357, 3359-3364. [CrossRef]

29. Domingo, V.; Prieto, C.; Silva, L.; Rodilla, J.M.L.; Quilez del Moral, J.F.; Barrero, A.F. Iodine, a mild reagent for the aromatization of terpenoids. J. Nat. Prod. 2016, 79, 831-837. [CrossRef]

30. Hou, L.; Ding, G.; Guo, B.; Wenhua, W.; Zhang, X.; Sun, Z.; Shi, X. New sesquiterpenoids and a diterpenoid from Alpinia oxyphylla. Molecules 2015, 20, 1551-1559. [CrossRef]

31. Kolev, J.N.; Zaengle, J.M.; Ravikumar, R.; Fasan, R. Enhancing the Efficiency and regioselectivity of P450 oxidation catalysts by unnatural amino acid mutagenesis. ChemBioChem 2014, 15, 1001-1010. [CrossRef]

32. Hu, H.; Bradley, S.A.; Krishnamurthy, K. Extending the limits of the selective 1D NOESY experiment with an improved selective TOCSY edited preparation function. J. Magn. Reson. 2004, 171, 201-206. [CrossRef]

33. Furusawa, M.; Hashimoto, T.; Noma, Y.; Asakawa, Y. Highly efficient production of nootkatone, the grapefruit aroma from valencene, by biotransformation. Chem. Pharm. Bull. (Tokyo). 2005, 53, 1513-1514. [CrossRef] [PubMed]

34. Rubottom, G.M.; Vazquez, M.A.; Pelegrina, D.R. Peracid oxidation of trimethylsilyl enol ethers: A facile $\alpha$-hydroxylation procedure. Tetrahedron Lett. 1974, 15, 4319-4322. [CrossRef]

35. Arseniyadis, S.; Ferreira, M.d.R.R.; Quílez del Moral, J.; Martín Hernando, J.I.; Potier, P.; Toupet, L. Studies towards the total synthesis of taxoids: a rapid entry into bicyclo[6.4.0]dodecane ring system. Part 1. Tetrahedron: Asymmetry 1998, 9, 4055-4071. [CrossRef]

36. Grieco, P.A.; Gilman, S.; Nishizawa, M. Organoselenium chemistry. A facile one-step synthesis of alkyl aryl selenides from alcohols. J. Org. Chem. 1976, 41, 1485-1486. [CrossRef]

37. Jordan, R.A.; Schulze, T.L.; Dolan, M.C. Efficacy of plant-derived and synthetic compounds on clothing as repellents against Ixodes scapularis and Amblyomma americanum (Acari: Ixodidae). J. Med. Entomol. 2012, 49, 101-106. [CrossRef] [PubMed]

38. Federal Laboratory Consortium for Technology Transfer (FLC) (2018) Formulation of nootkatone as repellent and pesticide products against mosquitoes and ticks. Available online: https://www.federallabs.org/ successes/success-stories/formulation-of-nootkatone-as-repellent-and-pesticide-products-against (accessed on 13 October 2019).

(C) 2019 by the authors. Licensee MDPI, Basel, Switzerland. This article is an open access article distributed under the terms and conditions of the Creative Commons Attribution (CC BY) license (http://creativecommons.org/licenses/by/4.0/). 